\title{
Field emission studies in vertical test and during cryomodule operation using precise $x$-ray mapping system
}

\author{
Hiroshi Sakai, ${ }^{1,2 *}$ Enrico Cenni, ${ }^{2, *}$ Kazuhiro Enami, ${ }^{1,2}$ Takaaki Furuya, ${ }^{1,2}$ Masaru Sawamura, ${ }^{3}$ \\ Kenji Shinoe, ${ }^{1, \dagger}$ and Kensei Umemori ${ }^{1,2}$ \\ ${ }^{1}$ High Energy Accelerator Research Organization (KEK), Tsukuba, Ibaraki 305-0801, Japan \\ ${ }^{2}$ The Graduate University for Advanced Studies (SOKENDAI), Hayama, Kanagawa 240-0115, Japan \\ ${ }^{3}$ National Institutes for Quantum and Radiological Science and Technology (QST), \\ Tokai, Naka, Ibaraki 319-1106, Japan
}

(Received 29 October 2018; published 21 February 2019)

\begin{abstract}
Field emission is one of the most serious issues in the degradation of superconducting cavity performance. However, surveying field emission sources during the cavity performance test (called the "vertical test") and cryomodule operation is difficult. Therefore, to precisely investigate electron emissions from the field emission source, we developed a diagnostic system for the energy recovery linac $1.3 \mathrm{GHz}$ nine-cell superconducting cavity for both vertical tests and the cryomodule operation. The developed system is comprised of two types of sensors: a carbon sensor and a $\mathrm{Si} p-i-n$ diode, that measure the temperature rise and the radiation produced by electron emissions, respectively. Rotating the sensor array around the cavity axis in the vertical test provides detailed information on the entire cavity surface. The precise $\mathrm{x}$-ray mapping profile measured by the system in the vertical test enables identification of the local emission source. This paper describes how the field emission source is identified in the vertical test from the standpoint of not only experimental results obtained with the newly developed rotating mapping system but also the detailed approach based on precise simulations. In addition, field emission studies with the optimized instrumentation have been extended to cryomodule tests, both standalone and in the beam line. These developments allow us to monitor changes in the cavity field emission signatures introduced by cryomodule assembly and beam operation.
\end{abstract}

DOI: 10.1103/PhysRevAccelBeams.22.022002

\section{INTRODUCTION TO FIELD EMISSION STUDIES}

Production of a high gradient field and high-current beam operation is a key requirement for particle acceleration. A superconducting cavity is one of the most promising technologies for accelerating particles owing to the advantage of near-zero electrical resistance of the accelerating cavity surface compared with a normal conducting cavity. For example, in high-energy physics, the linear collider is based on superconducting accelerators with a high gradient accelerating field [1]. On the other hand, free-electron lasers such as EURO-XFEL [2] and LCLS-II [3] and light sources based on the energy recovery

\footnotetext{
* Corresponding author.

hiroshi.sakai.phys@kek.jp

Deceased.

Present address: CEA/Universite Paris-Saclay, Saclay, France. enrico.cenni@cea.fr

Published by the American Physical Society under the terms of the Creative Commons Attribution 4.0 International license. Further distribution of this work must maintain attribution to the author(s) and the published article's title, journal citation, and DOI.
}

linac (ERL) [4] have also been designed and constructed as next-generation light sources with superconducting cavities. An ERL-based light source requires reliable high-current beam operation with a high gradient field generated by a superconducting cavity.

In the High Energy Accelerator Research Organization (KEK), a compact ERL (cERL) was constructed to study the feasibility of the future $3 \mathrm{GeV}$ ERL light source in 2009 $[5,6]$. The designed cERL consists of a high-brightness photoemission $500 \mathrm{kV}$ dc gun that can produce ultralow emittance beams with a high average current for an extended period [7], a drive-laser system with green $100 \mathrm{~W}$ power, injector superconducting cavities that can provide high rf power to beam up to $5 \mathrm{MeV}$ beam energy [8], superconducting cavities for a main linac having a high unloaded $Q$ of more than $1 \times 10^{10}$ with an accelerating gradient of more than $15 \mathrm{MV} / \mathrm{m}$ under the energy recovery condition [9], a recirculating loop for energy recovery and maintenance of the high-brightness beam quality, and the beam dump of the decelerated beam. It is important for main-linac superconducting cavities to not only achieve a high accelerating gradient $\left(E_{\mathrm{acc}}\right)$ of approximately $15 \mathrm{MV} / \mathrm{m}$ in beam operation but to also strongly damp higher-order modes (HOMs), because suppression of beam-breakup (BBU) 
instability due to the HOMs is one of the key issues for highcurrent operation of more than $100 \mathrm{~mA}$ at the ERL light source. In order to achieve the requirements of both a high gradient and strong damping of the HOMs, we specially designed a cavity for the main linac of cERL called the "KEK-ERL model-2 cavity" by optimizing the TESLA-type cavity. The design strategy of our ERL cavity, which includes a cryomodule, is described in the next section and in Ref. [9] in detail. This cavity, which is based on the $1.3 \mathrm{GHz}$ nine-cell elliptical cavity, has a large iris diameter of $80 \mathrm{~mm}$ with new cell shapes and enlarged beam pipes to allow propagation of the HOMs out of the beam pipe to the HOM dampers installed outside the nine-cell cavity in order to strongly suppress the HOMs. These result in a relatively high surface electric field at its iris region, which is a concern for field emission. Therefore, suppression of field emission is more essential for our cavity to operate stably than for the other existing superconducting cavities. This situation led us to initiate an intensive study of field emission and to develop an x-ray mapping system for investigation of the precise field emission profiles not only in vertical tests but also in cryomodule operation.

One of the causes of degradation of the superconducting cavity is thermal breakdown or field emission at a localized point on the cavity surface. Although temperature mapping is usually employed to investigate degradation of cavity performance, radiation mapping provides important additional information for identifying the field emission source, because the electrons emitted from the field emission source would hit the cavity surface, directly generating the radiation or $\mathrm{x}$ ray of the hit points. Therefore, it is important to obtain both the temperature and the $\mathrm{x}$-ray mapping in order to clarify the field emission source. The first investigation of the degradation of the cavity performance with both temperature and $\mathrm{x}$-ray mapping was performed using a $1.5 \mathrm{GHz}$ niobium single-cell cavity [10]. For a nine-cell cavity, there are two ways to obtain the temperature and $\mathrm{x}$-ray mapping: fixed mapping and rotating mapping. Fixed mapping was performed for the single-cell cavity by setting approximately 700 sensors on the cavity surface [11]. Fixed mapping for a nine-cell cavity requires more than 6000 sensors to obtain the precise mapping and many channels for data collection. In contrast, a rotating mapping system needs at most 100 sensors with a precise angular resolution determined by the gear ratio and stepping motor resolution. In addition, reducing the number of $p-i-n$ diodes reduces the number of current amplifiers needed to convert the photocurrent to voltage.

A fixed mapping system for the temperature and the $\mathrm{x}$ ray was recently developed for $1.3 \mathrm{GHz}$ nine-cell cavities [12]. Although this monitor helped us understand the thermal breakdown point and some defects in the inner surface, we could not identify the clear field emission source point because of the lack of angular resolution and the small number of $p-i-n$ diode monitors. On the TESLA nine-cell cavity, temperature mapping with the rotating mapping system was successfully measured [13]. Unfortunately, precise $\mathrm{x}$-ray mapping was not done for the TESLA ninecell cavity. In our ERL nine-cell cavity case, it is important to clarify that the newly designed cell shapes and enlarged beam pipes do not generate field emission within our requirements. Therefore, we decided to choose the rotating mapping system for our ERL nine-cell cavity to obtain good angle resolution with the reduced channels and to add x-ray sensors all over the cavity surface to investigate the field emission source. To evaluate the performance of this new rotating mapping system, we first applied to the $1.3 \mathrm{GHz}$ niobium single-cell cavity the same cell shape of the KEKERL model-2 cavity and performed the vertical tests [14]. The precise temperature and $\mathrm{x}$-ray mapping data were successfully measured [15]. These measured data indicated that the newly developed enlarged beam pipe called the eccentric fluted-beam pipe, which is explained in Sec. II, with the input and pickup port did not become a source of electron emission or a heating spot. Utilizing these results, we proceeded to fabricate the nine-cell KEK-ERL model-2 cavity and finally produced the cryomodule after the vertical tests.

Our goal is to suppress and eliminate the field emission effectively not only in a vertical test but also during cryomodule operation to ensure stable cavity performance. However, before this can be carried out, it is essential to deeply understand how the field emission is generated after the assembly work and during cryomodule operation. Thus, in this study, the field emission mechanism was first analyzed by using the x-ray rotating mapping system in the vertical test of our ERL nine-cell cavity. Furthermore, as continuous monitoring of the field emission properties from the vertical test to the cryomodule operation is important for the determination of how the field emission is generated, the development of an optimized field emission property monitoring system for this scenario is essential.

The remainder of this paper is organized as follows. Section II briefly outlines the main linac cavity and cryomodule of the cERL. Section III presents the experimental setup utilized for field emission studies and the measurement results of the vertical tests. Section IV compares the measurement results, including inspection of the inner cavity surface and the simulation results of field emission. Section V describes the experimental setup and analyzes the results obtained from the field emission studies for application to the cERL main linac cryomodule. The vertical test results and the cryomodule test results are systematically compared. Section VI concludes this paper.

\section{KEK-ERL MODEL-2 CAVITY AND ITS CRYOMODULE FOR MAIN LINAC AT CERL}

The TESLA cavity, which is designed for the linear collider project and the EURO-XFEL project, is a representative $L$-band nine-cell superconducting cavity. However, for high-current ERL operations of more than $100 \mathrm{~mA}$, its HOM damping capability is insufficient. Furthermore, the 


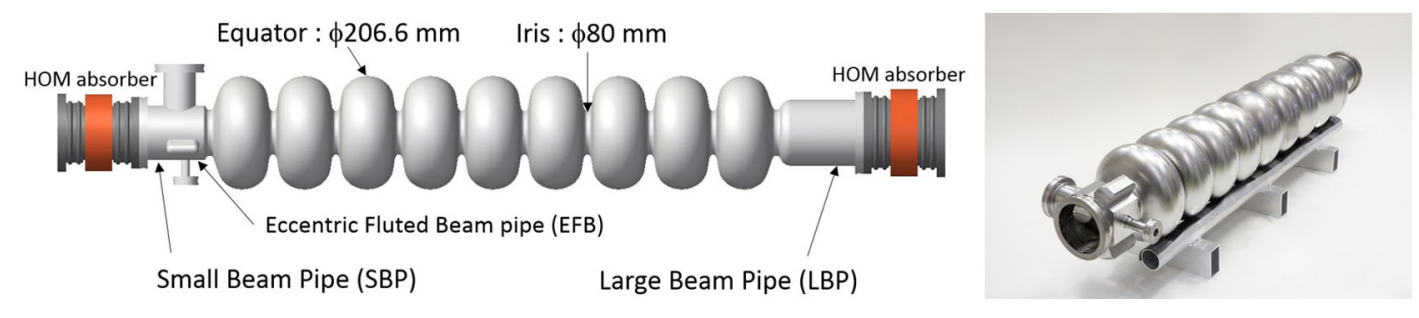

FIG. 1. Conceptual design (left) and picture (right) of the KEK-ERL model-2 cavity.

loop-type HOM couplers adopted for the TESLA cavity have a heating problem for continuous wave operation [16]. Therefore, we decided to develop a $1.3 \mathrm{GHz}$ superconducting cavity optimized for the ERL operations, especially for HOM damping.

Figure 1 shows the conceptual design and a picture of the KEK-ERL model- 2 cavity. The target accelerating gradient is more than $15 \mathrm{MV} / \mathrm{m}$, and the target unloaded $Q$ is more than $1 \times 10^{10}$ with an accelerating gradient of $15 \mathrm{MV} / \mathrm{m}$. Table I shows the main parameters of both our cavity and the TESLA cavity. The design strategy of our KEK-ERL model-2 cavity was reported in Ref. [9] in detail. Here, we briefly present the salient features of this cavity compared with the TESLA-type cavity. The first such feature is that the cavity employs enlarged beam pipes, with diameters of 100 [small beam pipe (SBP)] and $123 \mathrm{~mm}$ [large beam pipe (LBP)], to extract the HOMs from the cavity to the HOM absorbers. The second is the changing of the cell shapes and the iris diameter to $80 \mathrm{~mm}$ to reduce the impedances of the HOMs. The third is the application of the eccentricfluted beam pipe (EFB) to suppress quadrupole HOMs [17]. As a result, this design satisfies the $>100 \mathrm{~mA}$ current ERL operation required to avoid HOM-BBU instability [9]. However, the ratio of the peak electrical field on the surface divided by the accelerating field $\left(E_{\text {peak }} / E_{\text {acc }}\right)$ of our cavity is 1.5 times higher than that of the TESLA cavity. The highest electrical field mainly appears on the iris points of both nine-cell cavities.

A schematic diagram and a picture of the main-linac cryomodule are shown in Fig. 2. In this cryomodule, two

TABLE I. Parameters of the KEK-ERL model-2 cavity and TESLA cavity.

\begin{tabular}{lcc}
\hline \hline Parameters of nine-cell cavities & KEK-ERL model-2 & TESLA \\
\hline Resonant frequency $[\mathrm{MHz}]$ & 1300 & 1300 \\
Iris diameter [mm] & 80 & 70 \\
Equator diameter $[\mathrm{mm}]$ & 206.6 & 206.6 \\
Beam pipe diameter $[\mathrm{mm}]$ & $100 / 123(\mathrm{SBP} / \mathrm{LBP})$ & 78 \\
Shunt impedance per & 897 & 1030 \\
$\quad Q(R / Q)[\Omega]$ & & \\
Geometrical factor & 289 & 270 \\
$\quad\left(\Gamma=R_{s} \times Q_{0}\right)[\Omega]$ & 3.0 & 2.0 \\
$E_{\text {peak }} / E_{\text {acc }}$ & 42.5 & 42.6 \\
$H_{\text {peak }} / E_{\text {acc }}[\mathrm{Oe} /(\mathrm{MV} / \mathrm{m})]$ & & \\
\hline \hline
\end{tabular}

KEK-ERL model-2 cavities with helium jackets are mounted and surrounded by $5 \mathrm{~K}$ thermal shields, magnetic shields, and $80 \mathrm{~K}$ thermal shields. Two input couplers [18], two frequency tuners [19], and three HOM absorbers [20] are also mounted in the cryomodule. Finally, the cavities are cooled to $2 \mathrm{~K}$ during the cryomodule operation.

\section{FIELD EMISSION STUDIES IN VERTICAL TESTS}

We fabricated four KEK-ERL model-2 cavities. The first cavity, called the No. 1 ERL cavity, was fabricated to confirm that our fabrication technique for the niobium cavity and the surface treatment for this new shape would satisfy our requirements of a high unloaded $Q$ of more than $1 \times 10^{10}$ with an accelerating gradient greater than $15 \mathrm{MV} / \mathrm{m}$. The second cavity, called the No. 2 ERL cavity, was fabricated to achieve the mechanical properties required by the Japanese high-pressure vessel law by adding stiffener rings between the center cells and by welding titanium end plates between the beam pipes and the end cells. The third and fourth cavities, called the No. 3 and No. 4 ERL cavities, respectively, were fabricated to be installed into the cERL main linac cryomodule. In this section, we describe the field emission studies conducted with the No. 1 and No. 2 ERL cavities using the newly developed temperature and x-ray rotating mapping system. (The field emission studies conducted with the No. 3 and No. 4 ERL cavities are described in Sec. V together with the cryomodule operation.)

\section{A. Setup of the vertical test with temperature and $\mathrm{x}$-ray rotating mapping system [21]}

Figure 3 shows the setup for the vertical test with the KEK-ERL model-2 cavity using the rotating mapping system. A carbon resistor (Allen-Bradley, $50 \Omega$ ) and a Si $p$ - $i-n$ diode (HAMAMATSU, S5821-02) were set to measure the heat spot and the $x$ ray, respectively. Four carbon resistors per cell were set on the equator points at angles of $90^{\circ}$, as shown in Fig. 3. In addition, six carbon resistors, eight $\mathrm{Si} p-i-n$ diodes per cell, and one $p-i-n$ diode per iris were arranged along the cavity, as shown in Fig. 3 (right). A total of 90 carbon sensors and $82 p-i-n$ diodes were incorporated in this mapping system. All the carbon 

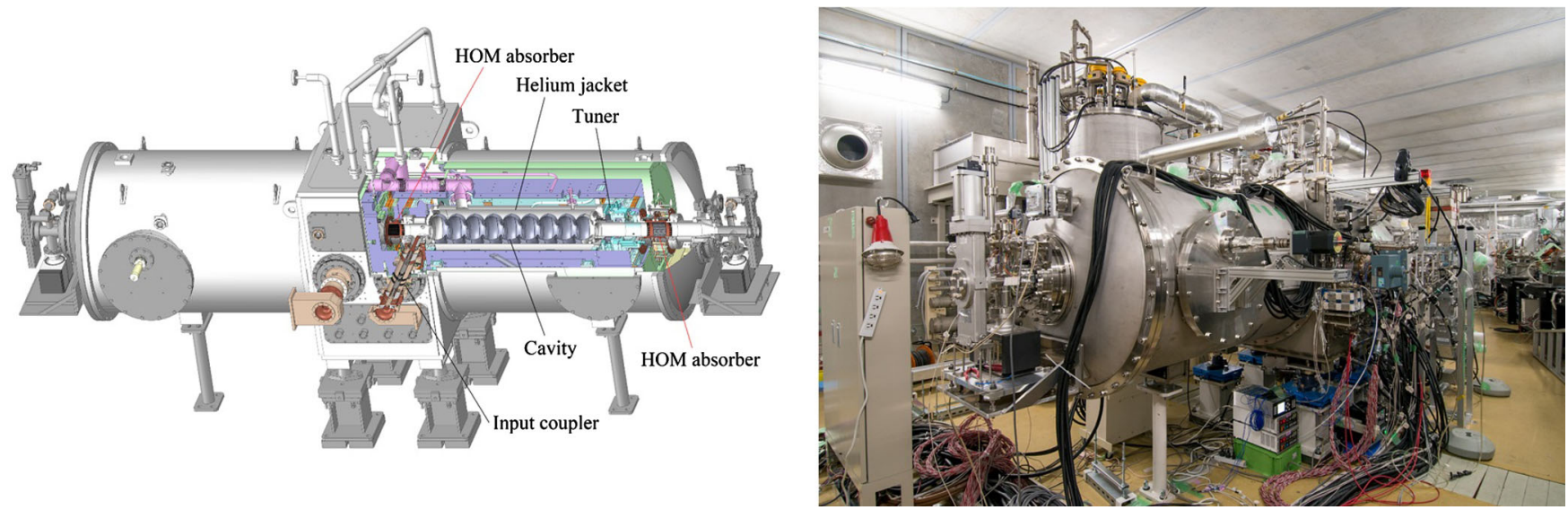

FIG. 2. Schematic diagram (left) and picture (right) of the main-linac cryomodule [6].
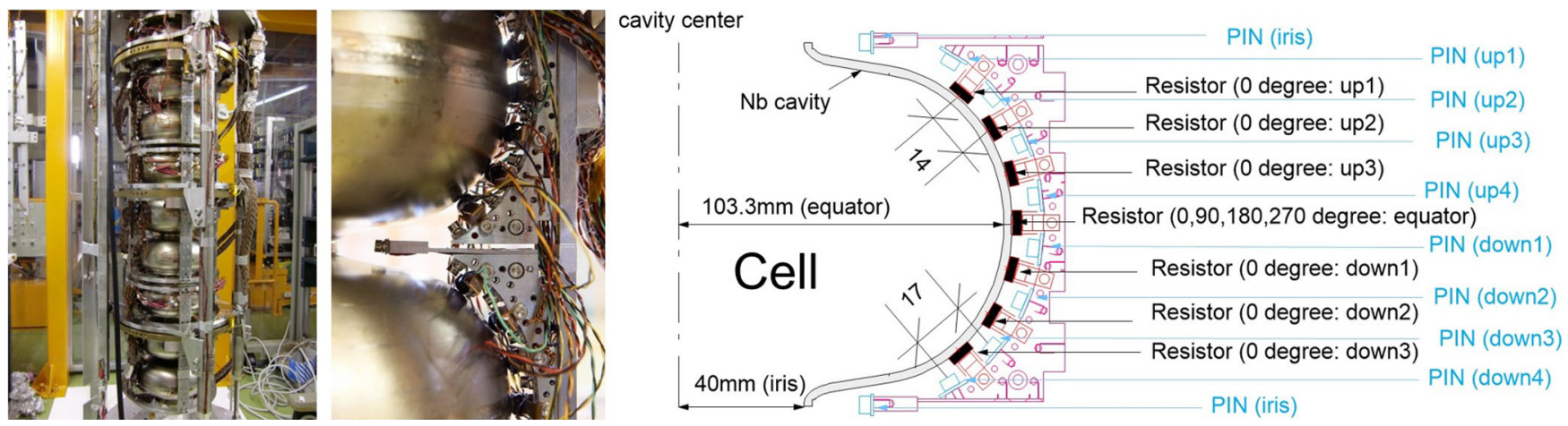

FIG. 3. Setup of the vertical test with the rotating mapping system of KEK-ERL model-2 cavity. (Left) Pictures of the overall mapping system and expanded view around the sensors. (Right) Sensor positions of the mapping system around one cell. Four carbon resistors per cell were set on the equator points every $90^{\circ}$. In addition, six carbon resistors (solid black), eight Si $p-i-n$ diodes per cell, and one $p$ - $i$ - $n$ diode per iris (light-blue) were arranged along the cavity at $0^{\circ}$ cavity angle. The $0^{\circ}$ cavity angle represents the initial point of the rotating mapping system.

resistors covered by the thermally conductive epoxy were pushed by the "pantograph-type" copper plate springs to obtain sufficient contact between the carbon resistors and the niobium cavity surface. All $p-i-n$ diodes and carbon resistors were fixed to the supports, which were divided into three parts. Each support with sensor arrays is equipped with a gear and turned around the cavity axis by rotating this gear via a motor. The resolution of the rotation angle was $0.5^{\circ}$. The generated current at the $p-i-n$ diode was converted to voltage by operational amplifiers. These data, including data from carbon resistors, $p-i-n$ diodes, power meters for the cavity rf measurement, and rotation angles, were collected by a real-time data acquisition system (YOKOGAWA, MX-100 and MW-100) every $0.5 \mathrm{~s}$. All carbon resistors were calibrated with a Si diode thermosensor (Lakeshore, DT-670 silicon diodes) under cooling from room temperature to $2 \mathrm{~K}$. The output voltages of all the $p-i-n$ diodes were checked with a white light from a light-emitting diode before cooling down in every vertical test.

\section{B. Measurement results of vertical tests of No. 1 ERL cavity with rotating mapping system}

The cavity surface treatments of the No. 1 ERL cavity are summarized in Table II. The cavity with $2.8 \mathrm{~mm}$ thickness was electropolished at first to remove the $130 \mu \mathrm{m}$ thickness of the inner surface of the cavity made of niobium (called EP1). Subsequently, the cavity was annealed at $750^{\circ} \mathrm{C}$ for $3 \mathrm{~h}$, and then $20 \mu \mathrm{m}$ of the inner surface of the cavity was removed by a second round of electropolishing (called EP2) after pretuning to obtain good field flatness of the cavity. Next, the cavity was washed with an ultrasonic bath and high-pressure ultrapure water [called high-pressure rinsing (HPR)] for more than $9 \mathrm{~h}$. The cavity was then moved into a class 10 (ISO class 4) cleanroom, and all the ancillary systems were assembled into the cavity in the class 10 cleanroom for the vertical test. After evacuation, the cavity was baked at $120^{\circ} \mathrm{C}$ for $48 \mathrm{~h}$.

Up to the third vertical test, we set only the fixed mapping system of 36 carbon resistors and 36 
TABLE II. No. 1 ERL cavity preparation and the history of vertical tests.

\begin{tabular}{lccc}
\hline \hline Vertical test number & Surface treatment before each vertical test & Maximum gradient & Limitation \\
\hline First test & EP1 $(130 \mu \mathrm{m})$, annealing, EP2 $(20 \mu \mathrm{m}), \mathrm{HPR}$, baking & $15 \mathrm{MV} / \mathrm{m}$ & Field emission \\
Second test & Baking & $15 \mathrm{MV} / \mathrm{m}$ & Field emission \\
Third test & HPR, baking & $15 \mathrm{MV} / \mathrm{m}$ & Field emission \\
Fourth test & EP2 $(50 \mu \mathrm{m}), \mathrm{HPR}$, baking & $17 \mathrm{MV} / \mathrm{m}$ & Field emission \\
Final test & $\mathrm{EP} 2(120 \mu \mathrm{m}), \mathrm{HPR}$, baking & $25 \mathrm{MV} / \mathrm{m}$ & Administrative limit \\
\hline \hline
\end{tabular}

$p-i-n$ diodes, which were set on the equator points every $90^{\circ}$. Unfortunately, in all three vertical tests, severe field emission limited the accelerating field to $15 \mathrm{MV} / \mathrm{m}$, even though we applied the additional surface treatment of HPR. In order to investigate the field emission phenomenon in detail, the rotating mapping system was newly applied from the fourth vertical test onward. Before the fourth vertical test, we applied electropolishing for $50 \mu \mathrm{m}$ removal of the inner surface of the cavity, HPR, and $120^{\circ} \mathrm{C}$ baking for $48 \mathrm{~h}$.

At first, some processing occurred owing to multipacting in the cells in the fourth vertical test. Figure 4 shows the measurement results of the accelerating field $\left(E_{\text {acc }}\right)$ and unloaded $Q\left(Q_{0}\right)$ of the last $\pi$-mode measurement ( $Q-E$ plot) during the fourth vertical test after processing. The radiation dose was also detected with another radiation monitor based on a Si diode (Hitachi Aloka Medical Ltd., MAR-782) set at the top flange of the cryostat at the vertical test stand (SBP side), as shown in Fig. 4. The radiation dose appeared at $8 \mathrm{MV} / \mathrm{m}$. Figure 5 shows the $\mathrm{x}$-ray mapping data under a $13.9 \mathrm{MV} / \mathrm{m}$ accelerating field of the $\pi$ mode

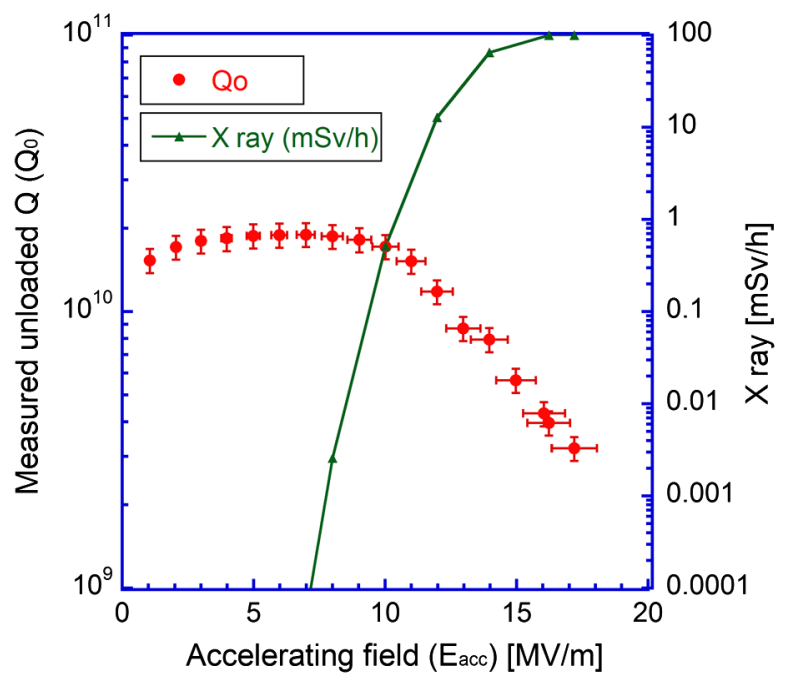

FIG. 4. Results of the fourth vertical test of the No. 1 ERL cavity. The horizontal axis shows $E_{\text {acc }}$ of the measured accelerating field. The left (right) vertical axis shows the measured unloaded $Q$ (measured x-ray on the top flange). The dynamic range of the radiation monitor is from 0.0001 to $100 \mathrm{mSv} / \mathrm{h}$. after processing. It took approximately $2.5 \mathrm{~min}$ to obtain the whole mapping all over the cavity with the mapping system. Unfortunately, we could not obtain the data of $p-i-n$ diodes set at the " $6-7$ iris" (the $\mathrm{m}-\mathrm{n}$ iris represents the iris between the mth and the nth cells) and "8cell down4," as indicated in Fig. 3, owing to the break of signal lines under the vertical test. Because the mapping system had a good angular resolution of $0.5^{\circ}$, which was determined by the data acquisition time, a clear $\mathrm{x}$-ray profile was observed. We note that no signals of the $\mathrm{x}$-ray mapping data were detected below a $10 \mathrm{MV} / \mathrm{m}$ accelerating field after processing.

The obtained x-ray profile was classified into two patterns in the fourth vertical test of the No. 1 ERL cavity as follows. A sharp x-ray spot was detected around the 8-9 iris at the cavity angle of approximately $330^{\circ}$. A broad spot was located at a cavity angle of approximately $150^{\circ}$, whose angle was on the opposite side of the sharp $\mathrm{x}$-ray spot, starting from the 5-6 iris to the iris of the first cell near the SBP. After the vertical test, the cavity was inspected with the inspection camera [22]. A niobium tip was found on the 8-9 iris at a $150^{\circ}$ cavity angle, as shown in Fig. 6. It was several tens of micrometers in height and several hundreds of micrometers in width with a sharp shape on the top. This location is just opposite the obtained sharp spot of x-ray mapping data, as shown in Fig. 5. We supposed that this tip might be a field emission source. After removing the tip with a local grinding machine [23] and applying the same surface treatment as in the fourth vertical test, we finally successfully achieved an accelerating field of $25 \mathrm{MV} / \mathrm{m}$ and satisfied the ERL specification of a high unloaded $Q$ of more than $1 \times 10^{10}$ with an accelerating gradient of $15 \mathrm{MV} / \mathrm{m}$ [24]. From these results, we conclude that the obtained $\mathrm{x}$-ray mapping data could indicate the field emission sources in detail. The comparison between the electron trajectory simulation and the measurement results is explained in Sec. IV.

\section{Comparison between temperature and x-ray mapping data under field emission}

Under the vertical tests of the No. 2 ERL cavity, we fully developed the temperature and $\mathrm{x}$-ray rotating mapping system. Thus, we obtained both the temperature and the $\mathrm{x}$-ray mapping data when the field emission occurred. 

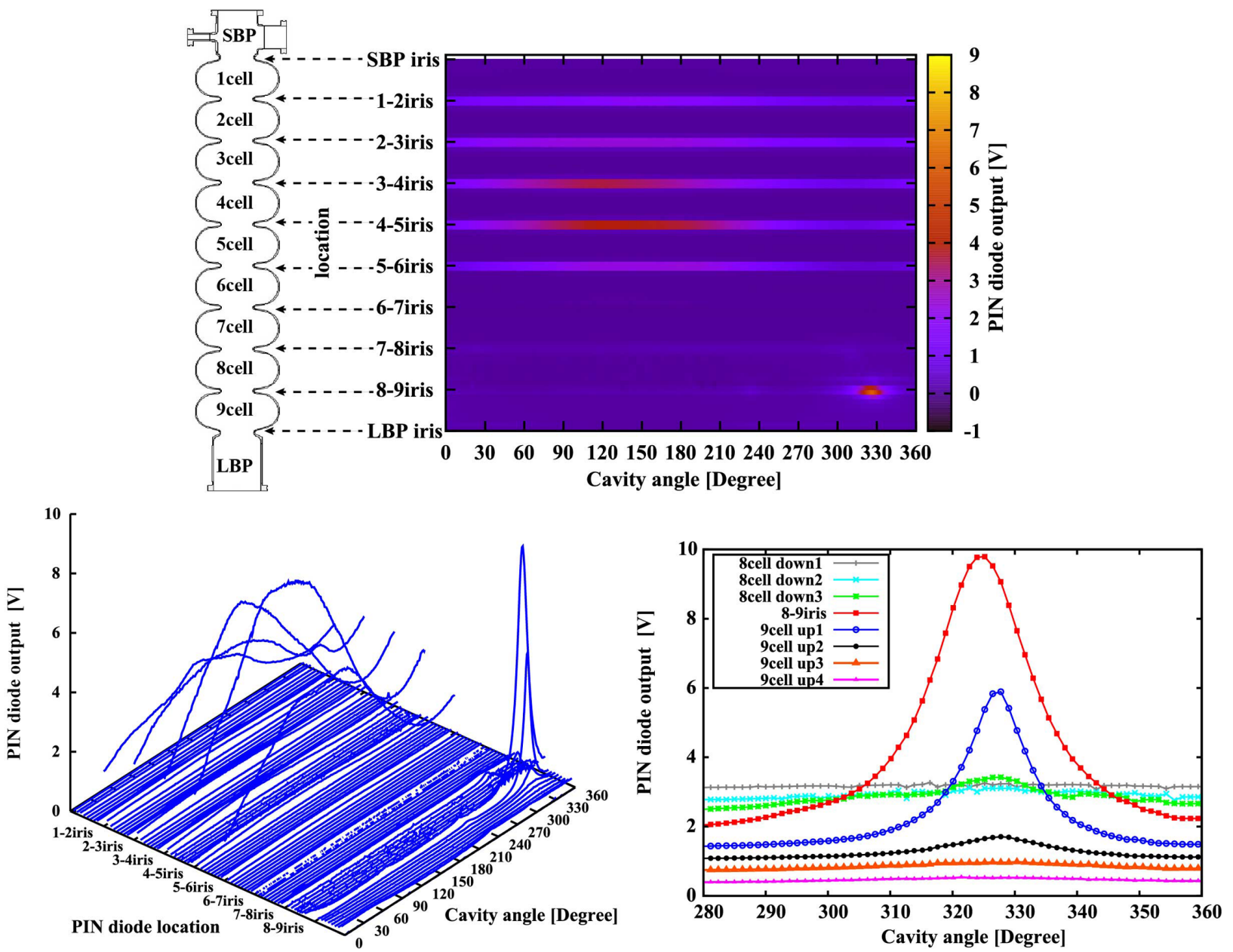

FIG. 5. (Top) X-ray mapping data in the fourth vertical test of the No. 1 ERL cavity under $13.9 \mathrm{MV} / \mathrm{m}$ accelerating field of the $\pi$ mode. The horizontal axis shows the rotating angle of the mapping system. The vertical axis shows the location of the $p$ - $i$ - $n$ diodes along the cavity axis, as shown in the left drawing. The color profile shows the voltages of the $p-i$ - $n$ diodes. The brighter color indicates a higher voltage, as shown in the right bar. Unfortunately, we could not obtain the data of $p$ - $i$ - $n$ diodes set at the "6-7 iris" and " 8 cell down 4 ," as indicated in Fig. 3, owing to the break of signal lines in the vertical test. (Bottom left) Three-dimensional plot of the x-ray mapping data as in the top figure. (Bottom right) Expanded view of the stacked x-ray mapping data near the sharp radiation spot on the eighth cell and the ninth cell around a $330^{\circ}$ cavity angle. The colored profiles of the figure show the stacked data of the sensors defined by Fig. 3 .

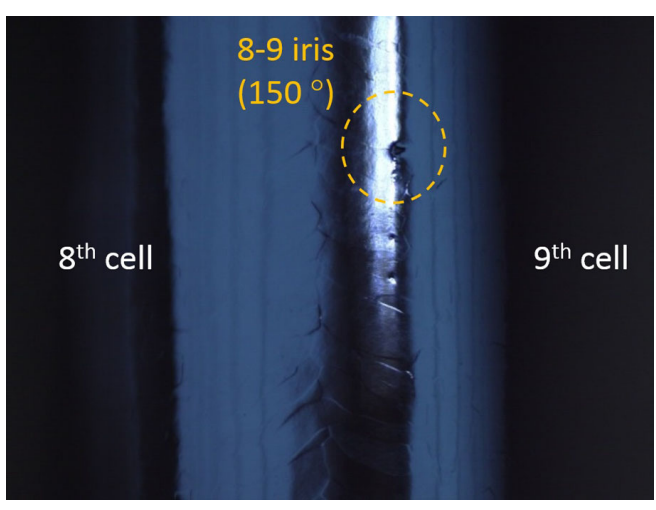

FIG. 6. Picture of the inner surface of the No. 1 ERL cavity taken with the inspection camera [22] after the fourth vertical test. The niobium tip was located at a $150^{\circ}$ cavity angle at the $8-9$ iris (the dotted orange circle).
Table III shows the history of the surface treatment of the No. 2 ERL cavity. The first surface treatment is almost the same as the No. 1 ERL cavity except for the first electropolished surface thickness. The cavity performance of the No. 2 ERL cavity also satisfied our ERL requirements in the vertical test. The detailed results of the vertical tests are described in Ref. [24]. Here, we show only the interesting results pertaining to the comparison between the temperature mapping and the $\mathrm{x}$-ray mapping data done in the final vertical test of the No. 2 ERL cavity.

Figure 7 shows the $Q-E$ plot of the $\pi$ mode and the measured radiation detected on the top flange of the cryostat during the final vertical test of the No. 2 ERL cavity. Radiation of the top flange appeared at $14 \mathrm{MV} / \mathrm{m}$, which is the starting point of the field emission. The temperature and $\mathrm{x}$-ray mapping data were collected 
TABLE III. No. 2 ERL cavity preparation and the history of vertical tests.

\begin{tabular}{|c|c|c|c|}
\hline Vertical test number & $\begin{array}{c}\text { Surface treatment before } \\
\text { each vertical test }\end{array}$ & Maximum gradient & Limitation and comments \\
\hline First test & $\begin{array}{l}\text { EP1 }(100 \mu \mathrm{m}), \text { annealing, } \\
\text { EP2 }(20 \mu \mathrm{m}), \text { HPR, baking }\end{array}$ & $23 \mathrm{MV} / \mathrm{m}$ & $\begin{array}{l}\text { Field emission and quench } \\
\text { turn-on event occurred }\end{array}$ \\
\hline Second test & EP2 $(20 \mu \mathrm{m})$, HPR, baking & $25 \mathrm{MV} / \mathrm{m}$ & Field emission and $Q$ decreased at low field \\
\hline Final test & Warm up & $25 \mathrm{MV} / \mathrm{m}$ & Field emission \\
\hline
\end{tabular}

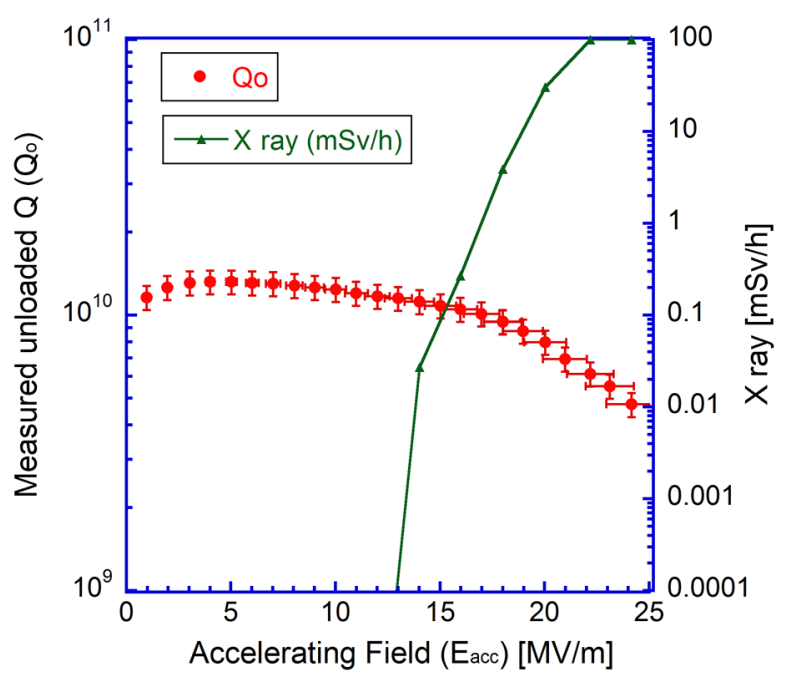

FIG. 7. Results of the final vertical test of the No. 2 ERL cavity. The horizontal axis shows $E_{\mathrm{acc}}$ of the measured accelerating field. The left (right) vertical axis shows the measured unloaded $Q$ (measured $\mathrm{x}$-ray on the top flange). The dynamic range of the radiation monitor is from 0.0001 to $100 \mathrm{mSv} / \mathrm{h}$. simultaneously during the final vertical test under a $23 \mathrm{MV} / \mathrm{m}$ accelerating field of the $\pi$ mode, as shown in Fig. 8. Some sensors at both end cells were removed compared with the vertical tests of the No. 1 ERL cavity, because the titanium end plates were prepared from the No. 2 ERL cavity and interfered with the sensors at both end cells. Unfortunately, in this vertical test, we could not obtain both the temperature and the X-ray mapping data from the first cell to the third cell because of gear failure. We obtained the whole mapping data from the fourth cell to the ninth cell including the 3-4 iris data. We had three clear sharp spots on the temperature mapping data in Fig. 8 (left). The first was the seventh cell near the 7-8 iris at a $50^{\circ}$ cavity angle, the second was the fourth cell near the 4-5 iris at a $155^{\circ}$ cavity angle, and the third was the sixth cell near the 6-7 iris at a $205^{\circ}$ cavity angle. We emphasize that the positions of the clear sharp peaks at the X-ray mapping data in Fig. 8 corresponded with the positions in the temperature mapping data. The x-ray mapping data in Fig. 8 (right) showed two other sharp peaks. One was located at the 3-4 iris at a $135^{\circ}$ cavity angle, and another was at the 4-5 iris at a $225^{\circ}$ cavity angle. Unlike the No. 1 ERL cavity, the No. 2 ERL cavity had stiffener rings welded at all iris positions. The stiffener ring had small slits to fill helium on the iris
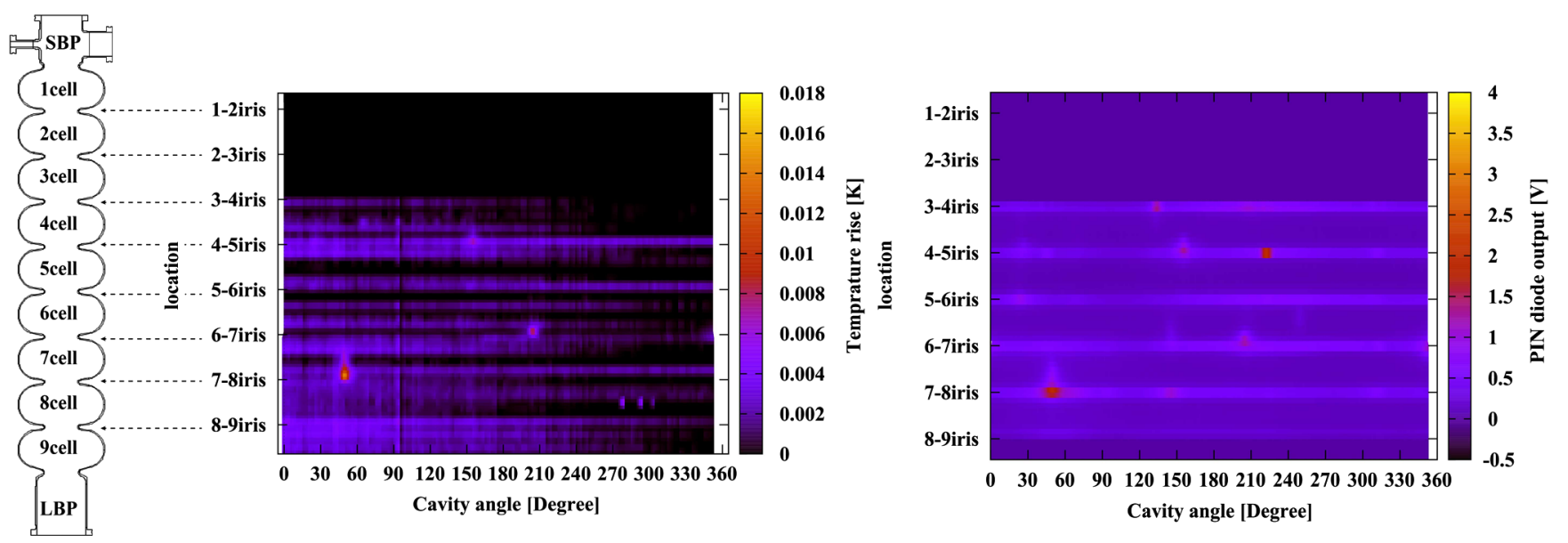

FIG. 8. Temperature mapping data (left) and x-ray mapping data (right) obtained in the final vertical test of the No. 2 ERL cavity at a $23 \mathrm{MV} / \mathrm{m}$ accelerating field. Both horizontal axes of the figures show the rotating angle of the mapping system. Both vertical axes of the figures show the location of the temperature sensors and $p-i-n$ diodes along the cavity axis. The color profile shows the voltages of the $p-i-n$ diodes (temperature rise) on the right (left) figure, respectively. The brighter color shows a higher voltage (temperature rise), as shown in the right bar of the right (left) figure. 

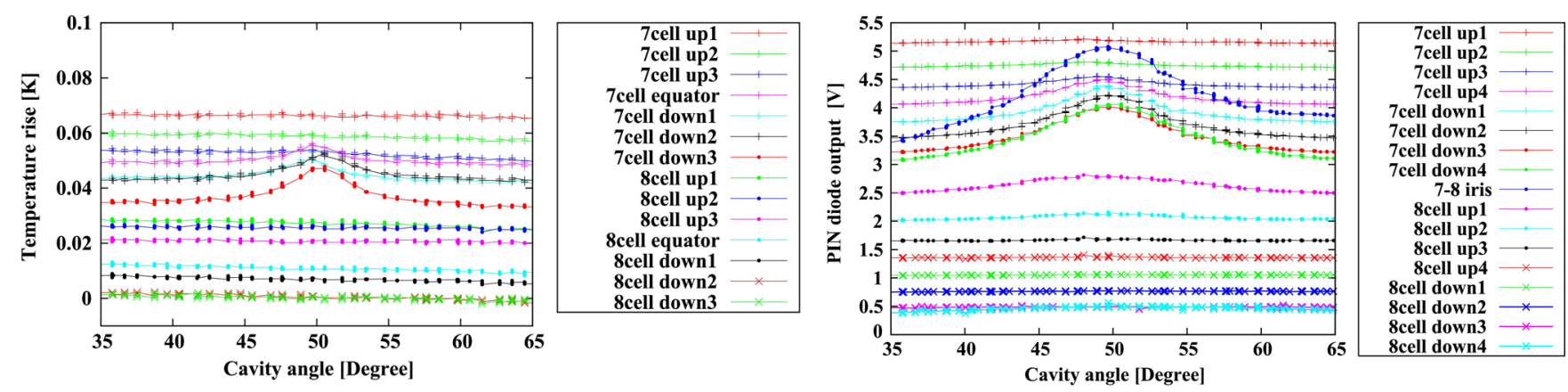

FIG. 9. Expanded views of the stacked temperature mapping data (left) and the stacked x-ray mapping data (right) near the sharp radiation spot on the seventh cell and the eighth cell at a cavity angle of approximately $50^{\circ}$. The colored profiles of both figures show the stacked data of the sensors defined by Fig. 3 .

points every $90^{\circ}$, whose locations were the same as the positions at $45^{\circ}, 135^{\circ}, 225^{\circ}$, and $315^{\circ}$ cavity angles on the mapping data. In order to compensate for the attenuation with and without the slits during the x-ray mapping measurement, we installed additional niobium pieces having the same thickness as the stiffener ring into all slits every $90^{\circ}$. Unfortunately, we found after this vertical test that the niobium pieces set at the $3-4$ iris at a $135^{\circ}$ cavity angle and at the $4-5$ iris at a $225^{\circ}$ cavity angle had fallen away. The large sharp $\mathrm{x}$-ray peaks at the 3-4 iris at a $135^{\circ}$ cavity angle and at the $4-5$ iris at a $225^{\circ}$ cavity angle were due to the absence of the niobium pieces. Because these two peaks are not real spots and are different in nature from the previous three sharp spots, the three brightest sharp spots on the temperature mapping data perfectly corresponded with the three brightest sharp spots on the $\mathrm{x}$-ray mapping data in Fig. 8.

We focused on the brightest spot located near the seventh cell at a $50^{\circ}$ cavity angle on both the temperature and the x-ray mapping data, as shown in Fig. 8, in order to investigate the nature of this sharp spot in detail. Figure 9 shows the expanded temperature and x-ray mapping data from the seventh cell to the eighth cell around a $50^{\circ}$ cavity angle. The peaks mainly appeared in the seventh cell including the 7-8 iris in both the temperature and $x$-ray mapping data in Fig. 9. In order to observe the dependence on the accelerating field, the sensor array was fixed to the peak position at a $50^{\circ}$ cavity angle. Figure 10 shows the data obtained with temperature sensors and x-ray sensors on the sharp spot of the seventh and the eighth cells. When the accelerating field almost reached $25 \mathrm{MV} / \mathrm{m}$, sudden temperature rises were observed at the seventh cell side as shown in Fig. 10 (left); the quench occurred. Before the quench occurred at $25 \mathrm{MV} / \mathrm{m}$, x-ray signals increased exponentially with the accelerating field; this is the typical phenomenon of field emission. We note that the temperature increased on the sharp spot of the seventh cell side as the $\mathrm{x}$-ray signals detected by the $p-i-n$ diodes set
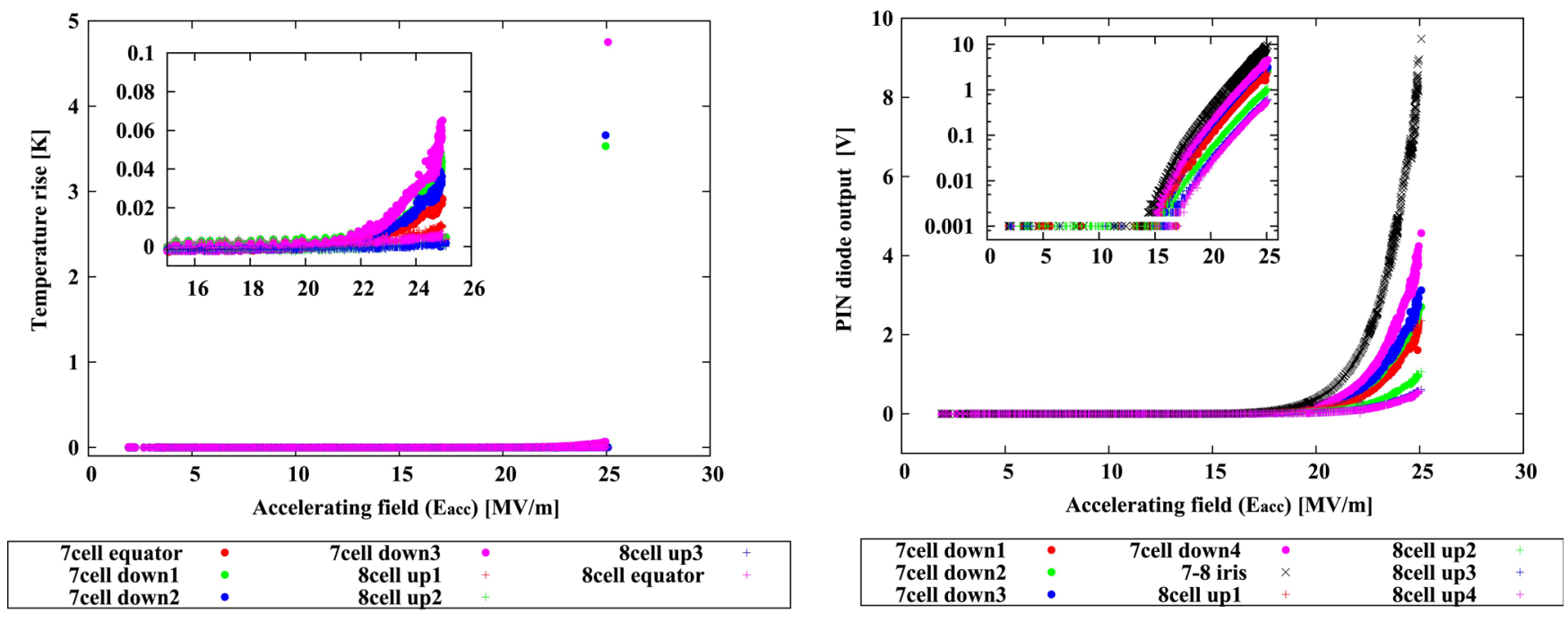

FIG. 10. Temperature rise (left) and $p-i-n$ diode output (right) as a function of the accelerating field. The inner figure of the left figure shows the expanded view around the quench field. The inner figure of the right figure shows the plot in which the vertical axis is logarithmically represented. All data near the seventh cell and the eighth cell were taken at a $50^{\circ}$ cavity angle. 


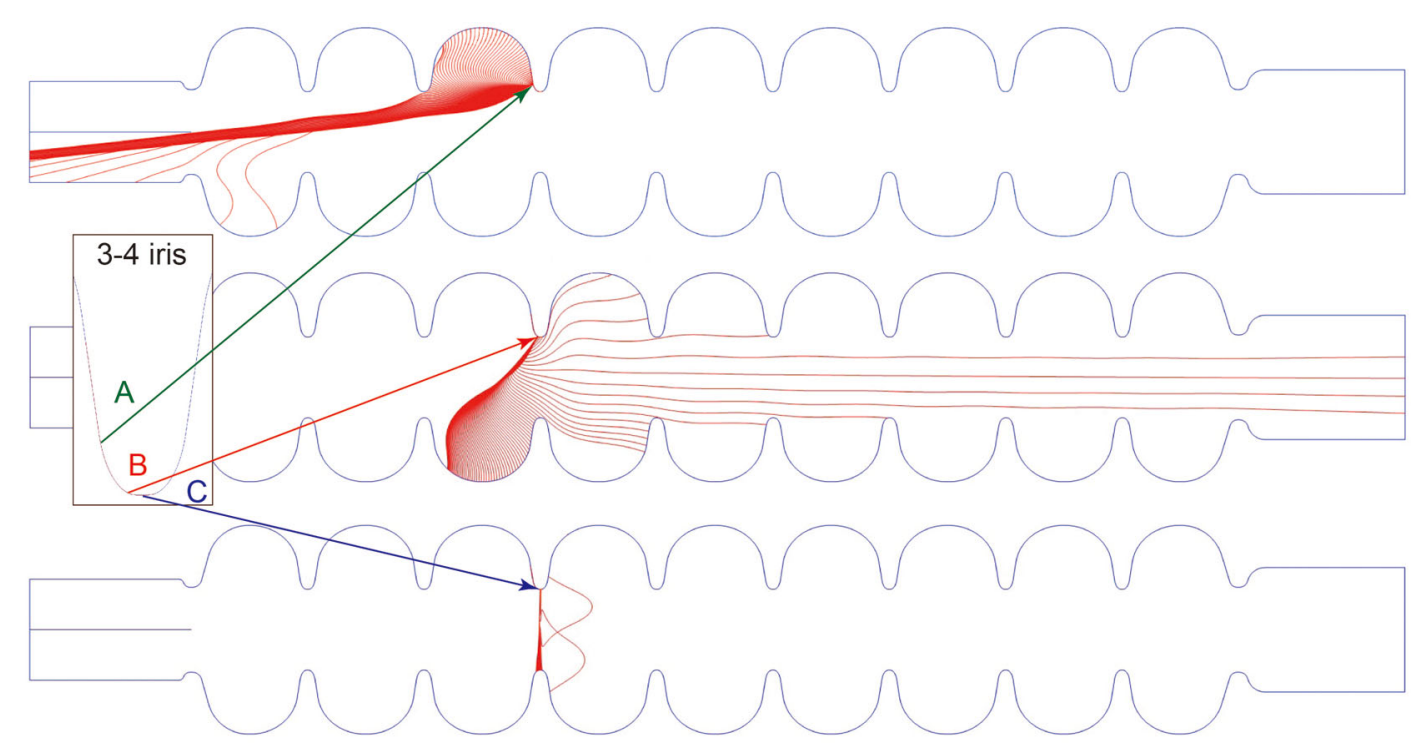

FIG. 11. Typical examples of electron trajectories from field emission sources near the iris position of the KEK-ERL model-2 cavity under an accelerating field of $15 \mathrm{MV} / \mathrm{m}$ of the $\pi$ mode. The emitter positions of $\mathrm{A}, \mathrm{B}$, and $\mathrm{C}$ are located $6.8,3$, and $0 \mathrm{~mm}$ from the iris center along the cavity axis, respectively.

around the seventh cell side including the 7-8 iris area increased. Judging from these measurement results shown in Figs. 7-10, we can conclude that the emitted electron from a field emission source hits this local point of the inner cavity surface and that this increased both $\mathrm{x}$-ray signals and the temperature at this local spot where the quench occurred. All sharp x-ray spots of the x-ray mapping data in Fig. 8 could be considered as the results of the impact of the electrons generated by the field emission.

\section{DISCUSSION OF THE FIELD EMISSION STUDIES IN VERTICAL TESTS}

From the field emission studies of the two KEK-ERL model-2 cavities with the temperature and $\mathrm{x}$-ray rotating mapping system, we obtained clear x-ray profiles when field emission occurred. We obtained two radiation patterns: the sharp spot and the broad spot. We also clarified that the x-ray spot was produced by electrons generated by field emission. In order to fully understand why electrons produced by field emission created these $\mathrm{x}$-ray spots as shown in Fig. 5, we calculated the electron trajectories on the basis of $\mathrm{rf}$ simulation including the multipacting simulation code $[25,26]$ with the KEK-ERL model-2 cavity shape by assuming that one field emission source would be located at the position where the tip was detected with the inspection camera, as shown in Fig. 6 [27,28]. We would like to, finally, identify the field emission source through a deep understanding of field emission by using these simulations whenever we obtained clear profiles of the $\mathrm{x}-$ ray mapping data. Before investigating the field emission source by field emission simulation, we first show the calculation procedure of field emission and give typical examples of electron trajectories produced by different field emission sources of the KEK-ERL model-2 cavities in this section.

\section{A. Electron trajectory simulation with different field emission sources of KEK-ERL model-2 cavity}

The field emission current is expressed by the wellknown "Fowler-Nordheim" (FN) equation as follows [29]:

$$
\begin{aligned}
I_{\mathrm{FE}}= & \frac{A_{e} A_{\mathrm{FN}}\left(\beta_{\mathrm{FN}} \cdot E_{\mathrm{surf}} \cdot \sin \theta\right)^{2}}{\Phi} \\
& \cdot \exp \left(-\frac{B_{\mathrm{FN}} \cdot \Phi^{1.5}}{\beta_{\mathrm{FN}} \cdot E_{\mathrm{surf}} \cdot \sin \theta}\right) \quad[A],
\end{aligned}
$$

where $I_{\mathrm{FE}}$ is the current due to field emission, $E_{\mathrm{surf}} \sin (\theta)$ is the electric rf field on the inner cavity surface in $\mathrm{V} / \mathrm{m}$ with the $\operatorname{rf}$ phase $\theta, \Phi$ is the niobium working function of magnitude $4.3 \mathrm{eV}, \beta_{\mathrm{FN}}$ is the field enhancement factor due to the asperity of the emission source, and $A_{\mathrm{FN}}$ and $B_{\mathrm{FN}}$ are constants that equal $1.54 \times 10^{-6}\left[\mathrm{AeV} \mathrm{V}^{-2}\right]$ and $6.83 \times 10^{9}\left[\mathrm{eV}^{-1.5} \mathrm{~V} \mathrm{~m}^{-1}\right]$, respectively [30]. $A_{e}$ is the emitter area of the inner surface of the cavity.

Figure 11 shows typical examples of electron trajectories from different field emission sources near the iris position. The complete trajectories of the rf phases are plotted at the intervals of $1^{\circ} \mathrm{rf}$ phase. We found that the calculated electron trajectories were very sensitive to the source position near the iris. The emitted electrons from the emitter position of " $C$," as shown in Fig. 11, move and hit the opposite iris. Then, these electrons do not pass through the cavity anymore. The electrons from the emitter position of " $A$," as shown in Fig. 11, are accelerated in the 
same direction as the initial electron direction and finally reach the beam pipe end. On the other hand, the electrons from the emitter position of " $B$," as shown in Fig. 11, are accelerated in the opposite direction from the initial electron direction on the surface as the rf field changes sinusoidally. In these cases, the emitted electrons with a small kinetic energy are first accelerated in the same direction, after which these electrons return and are finally accelerated backward as the rf field changes sinusoidally. We noted that the trajectories did not depend on the $\beta_{\mathrm{FN}}$ at the source point in Fig. 11. After our detailed simulation, the dependence of these trajectories was recently also simulated for the TESLA cavity shape, and the results were similar to ours [31]. The measurements of the x-ray profile with the fixed sensors at iris positions in the vertical test with the TESLA cavity were similar to our results [31]. We note that all electrons in one rf cycle are trapped within the same cell and are not accelerated to either beam pipe when the emitter position is closer to the equator than the position of $\mathrm{A}$. With these simulations of the emitter position dependence, we found that emitters closer to the iris are more dangerous, because they can induce higher-power electrons with higher current owing to the higher peak field and higher acceleration along the cavity axis.

\section{B. Comparison between $x$-ray mapping data in vertical tests and field emission simulation [28]}

Figure 12 (bottom) shows the electron trajectories under an accelerating field of $13.9 \mathrm{MV} / \mathrm{m}$ of the $\pi$ mode with different $\mathrm{rf}$ phases obtained by the field emission calculation based on the rf simulation including the multipacting simulation code when the emission source, where the tip was observed as shown in Fig. 6, is assumed to be located at a $1 \mathrm{~mm}$ distance on the LBP side from the 8-9 iris center at a $150^{\circ}$ cavity angle. We also show the impact energies of electrons from field emission on the surface along a $330^{\circ}$ cavity angle (the opposite side of the $150^{\circ}$ cavity angle) including the end cap of the SBP side and iris positions (blue dots) along the cavity axis in Fig. 12 (top). We found that some emitted electrons accelerated up to more than $8 \mathrm{MeV}$ to the SBP side as indicated by the pink dotted circles in Fig. 12. This is one of the reasons why the radiation was detected at the low cavity gradient field on the cryostat top flange far from the SBP end cap, as shown in Fig. 4.

The power landing on the cavity surface with the rf phase is expressed as follows:

$$
P_{\mathrm{FE}}=I_{\mathrm{FE}} \cdot V_{\text {impact }} \quad[\mathrm{W}],
$$

where $I_{\mathrm{FE}}$ is the current due to the field emission as shown in Eq. (1) and $V_{\text {impact }}$ is the accelerating voltage from the emitter position to the landing point on the cavity surface. The geometrical parameters of $\left(\beta_{\mathrm{FN}}, A_{e}\right)$ of the emitter

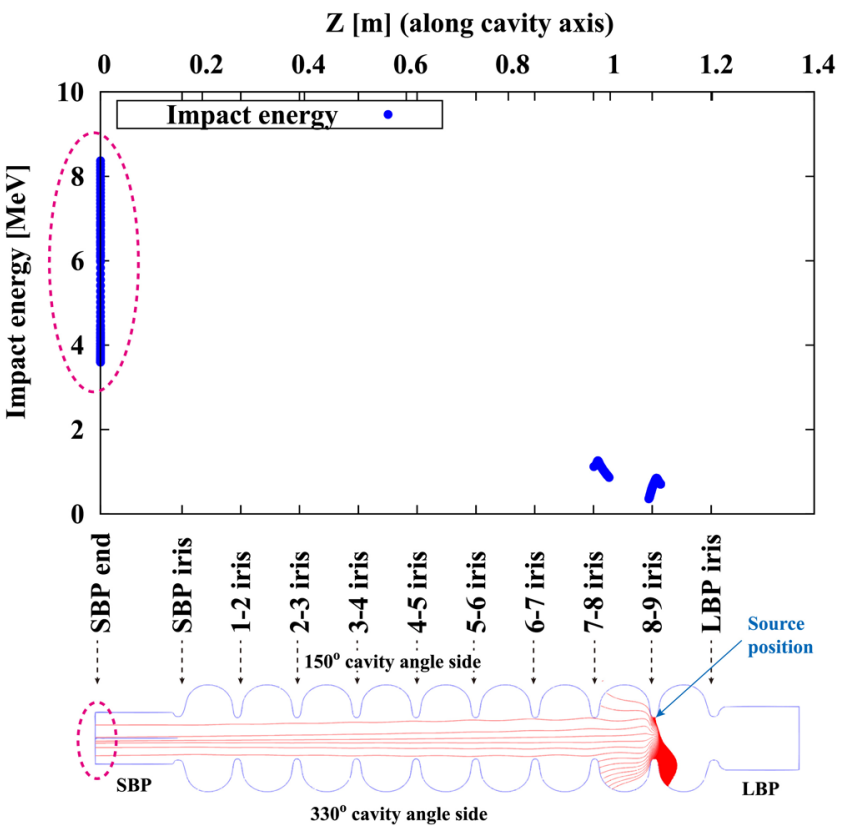

FIG. 12. (Bottom) Simulated electron trajectories (red lines) from the field emission source for the KEK-ERL model-2 cavity under an accelerating field of $13.9 \mathrm{MV} / \mathrm{m}$ of the $\pi$ mode for every $0.5^{\circ} \mathrm{rf}$ phase. The emitter is located at a $1 \mathrm{~mm}$ distance on the LBP side from the 8-9 iris center. (Top) The impact energies (blue dots) from the simulated electron trajectories inside the cavity along a $330^{\circ}$ cavity angle (opposite the emitter location) for every $0.05^{\circ} \mathrm{rf}$ phase. The horizontal (vertical) axis shows the length along the cavity axis with iris and SBP end positions (the impact energies of electrons on the cavity surface including the end cap), respectively. The impact energies surrounded by the pink dotted area reached the SBP end position denoted by the pink dotted area in the bottom figure.

could be estimated by using a best fit from the comparison between the power of $P_{\mathrm{FE}}$ obtained by Eq. (2) and the measured dissipated power from the $Q-E$ plot in Fig. 4 if we assume that the emitted electrons come from only one field emission source and their total energy is deposited in the cavity wall. As a result, the enhancement factor of $\beta_{\mathrm{FN}}$ and the emitter area of $A_{e}$ are estimated to be approximately 282 and $6 \times 10^{-16} \mathrm{~m}^{2}$, respectively [28]. We assume that the tip found with the inspection camera as shown in Fig. 6 is the source of the field emission, because the cavity performance described in Sec. III B improved after the removal of this tip. It is noted that $\beta_{\mathrm{FN}}$ should be estimated by the microstructure of the emitter [32]. However, unfortunately, the inspection camera does not have adequate resolution to identify the microstructure of this tip. We start the calculation for the power of $P_{\mathrm{FE}}$ with this tip as the source of the field emission with 282 of $\beta_{\mathrm{FN}}$ and $6 \times 10^{-16} \mathrm{~m}^{2}$ of $A_{e}$. In this calculation, we focus on the understanding of the radiation distribution compared with the absolute value of $P_{\mathrm{FE}}$.

Figure 13 shows the power landing on the cavity surface from the simulated electron trajectories under the same 


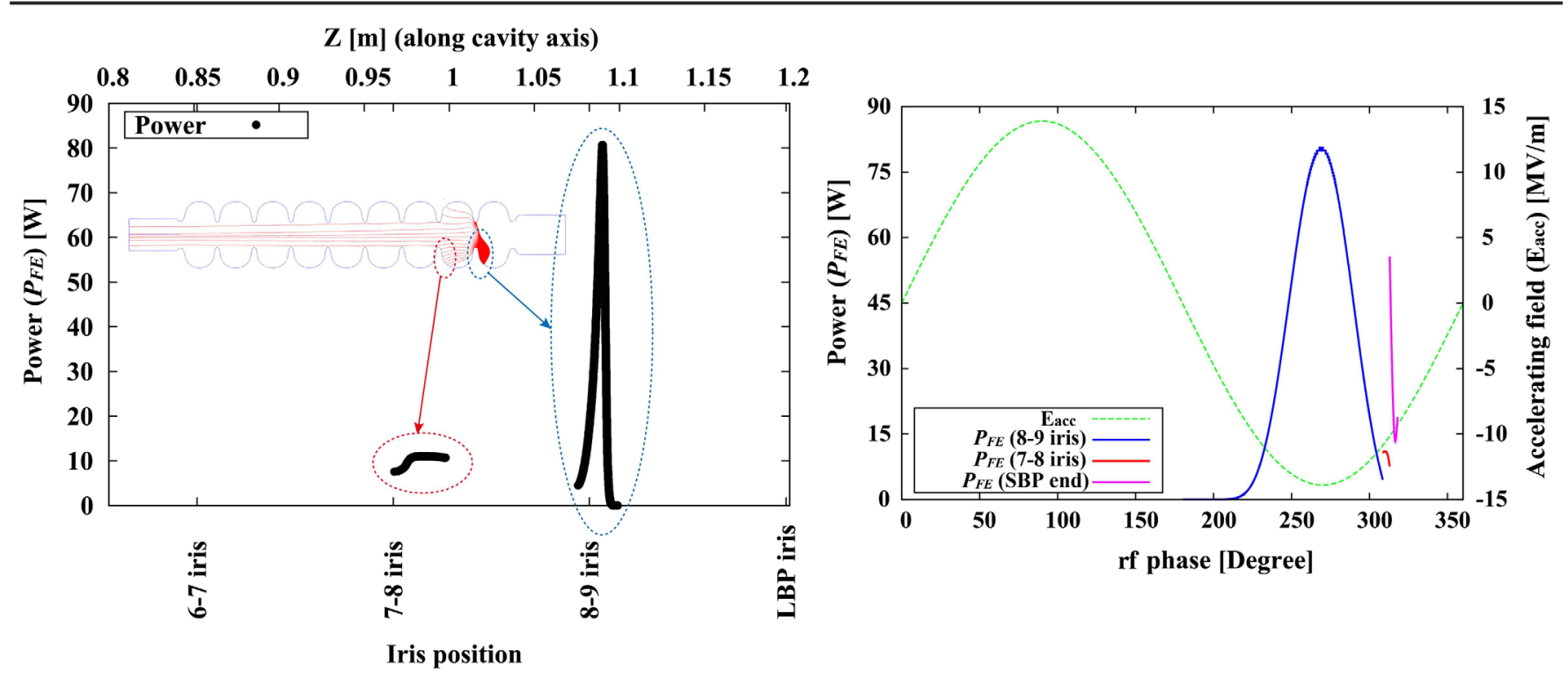

FIG. 13. (Left) Power landing on the cavity surface $\left(P_{\mathrm{FE}}\right)$ of the seventh cell, eighth cell, and ninth cell from the electron trajectories under the same conditions as shown in Fig. 12. The horizontal (vertical) axis shows the length along the cavity axis with iris positions [the power of $P_{\mathrm{FE}}$ calculated by . (2)], respectively. (Right) Power of $P_{\mathrm{FE}}$ as a function of the rf phase $\theta$. The horizontal (vertical) axis shows the rf phase (the power of $P_{\mathrm{FE}}$ and $E_{\mathrm{acc}}$ ), respectively. The blue line (red line) in Fig. 13 (right) shows the power landing near the 8-9 iris (7-8 iris), and the pink line shows the power on the SBP end. The green dotted line shows the accelerating field.

conditions as shown in Fig. 12. The power distribution from the seventh cell to the ninth cell on the cavity surface at a $330^{\circ}$ cavity angle is plotted along the cavity axis with the iris locations in Fig. 13 (left). A large sharp peak of power is found near the 8-9 iris as indicated by the blue dotted circles in Fig. 13 (left). The location of the power peak agrees well with the measured sharp spot of the x-ray mapping data shown in Fig. 5. Figure 13 (right) shows the power distribution as a function of the $\mathrm{rf}$ phase $\theta$. The blue line (red line) in Fig. 13 (right) shows the power landing near the 8-9 iris (7-8 iris), and the pink line shows the power on the SBP end. The electrons from the field emission are emitted only between the $180^{\circ} \mathrm{rf}$ phase and the $360^{\circ} \mathrm{rf}$ phase. The peak power of $P_{\mathrm{FE}}$ is estimated to be approximately $80 \mathrm{~W}$ at the $270^{\circ} \mathrm{rf}$ phase. From Fig. 13 (right), most of the power is distributed near the 8-9 iris and deposited on niobium in accordance with the calculation of the Electron Gamma Shower Version 5 (EGS5) simulation code [33], which calculates the interaction between the particles and the material of the cavity surface, niobium. The total deposited power is calculated from the average of $P_{\mathrm{FE}}$ and works out to $11 \mathrm{~W}$, which is almost consistent with the dissipated power from the $Q-E$ plot in Fig. 4. The transmitted signals through niobium of thickness $2.5 \mathrm{~mm}$ are detected as photons, which are produced by the hitting electrons with less than $1 \mathrm{MeV}$ kinetic energy via the interaction with niobium, from the calculation of the EGS5 simulation code. The transmitted power is less than $0.7 \%$ of the power of the hitting electrons. These photons spread out with the Gaussian shape at a scattering angle of approximately $30^{\circ}$ from the hitting point.
However, $p-i-n$ diodes were set closer to the niobium surface than several millimeters. Therefore, we were able to detect the $\mathrm{x}$-ray signals with a Gaussian shape of less than a $10^{\circ}$ cavity angle rms, which almost agrees with the spread of our local sharp x-ray profile, as shown in Fig. 5. From these simulations, we concluded that the electrons due to the field emission hit the opposite side of the same iris position and produced a sharp spot in the x-ray mapping data.

We note that this sharp spot was observed during the other passband mode measurement of the $7 / 9 \pi$ mode on the same fourth vertical test of the No. 1 ERL cavity, as shown in Fig. 14 [21]. The clear sharp spot at the $7 / 9 \pi$ mode was also observed at the same position as the sharp spot of the $\pi$ mode. However, the broad spot was not observed at the $7 / 9 \pi$ mode, unlike the observation with the $\pi$ mode. In order to explain the similarity and difference between the $\pi$ mode and the $7 / 9 \pi$ mode, the same field emission simulation was carried out with the rf field of the $7 / 9 \pi$ mode. The simulation result of the $7 / 9 \pi$-mode electron trajectories is shown in Fig. 14 (left) with the same emitter location under $13.1 \mathrm{MV} / \mathrm{m}$ of the accelerating field at the end cell. Most of the $7 / 9 \pi$-mode electrons due to the field emission hit the opposite side at the same 8-9 iris, because the end cell (ninth cell) fields of the $7 / 9 \pi$ mode and the $\pi$ mode are almost the same. The simulation results can also explain the appearance of the sharp spot in the $7 / 9 \pi$ mode. On the other hand, we found that the electrons do not accelerate to the SBP end in the $7 / 9 \pi$ mode. This is because the $7 / 9 \pi$ mode is not the accelerating mode of this cavity. It is expected that the electrons accelerated to the SBP side at the $\pi$ mode may contribute 


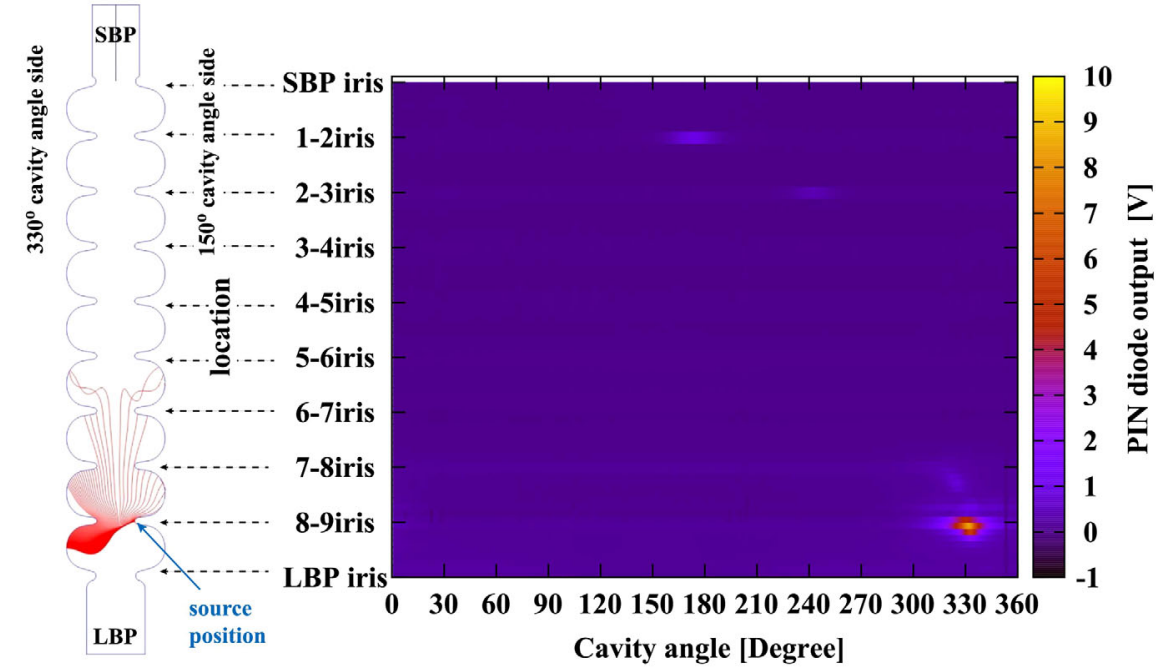

FIG. 14. X-ray mapping data in the fourth vertical test of the No. 1 ERL cavity under a $13.1 \mathrm{MV} / \mathrm{m}$ accelerating field at the end cell of the $7 / 9 \pi$ mode. The horizontal axis shows the rotating angle of the mapping system. The vertical axis shows the location of the $p-i-n$ diodes along the cavity axis. The color profile shows the voltages of the $p-i$ - $n$ diodes. The simulated electron trajectories (red lines) for every $1^{\circ} \mathrm{rf}$ phase from the same emitter location as shown in Fig. 12 with the $7 / 9 \pi$ mode under a $13.1 \mathrm{MV} / \mathrm{m}$ accelerating field at the end cell are shown on the left side.

to the creation of the broad spot at the $\pi$ mode as shown in Fig. 5.

By calculating the electron trajectories of the $\pi$ mode with a smaller phase step than that of the $0.05^{\circ} \mathrm{rf}$ phase, we found that some electron trajectories hit the 6-7, 5-6, and 4-5 irises with more than $2 \mathrm{MeV}$ kinetic energies within the phase step of the $0.05^{\circ} \mathrm{rf}$ phase. We constructed a model of the KEK-ERL model-2 cavity in the EGS5 simulation code and input incident electrons with more than $2 \mathrm{MeV}$ kinetic energies at the above iris positions. The EGS5 simulation results showed that most of the electrons were reflected at the iris position and scattered toward the opposite side while retaining their kinetic energies when the electrons were injected to the niobium of thickness $2.5 \mathrm{~mm}$ with an incident angle of less than $10^{\circ}$. We note that both the peak and the spread of the broad spot in Fig. 5 are very similar to the simulation results of the reflected electron's distribution created by this EGS5 simulation. Detailed explanations and the results of the EGS5 simulation with this model are given in Ref. [28].

In addition, the diode current of the $\mathrm{Si} p-i-n$ diode (HAMAMATSU, S5821-02) was calculated with the EGS5 simulation by changing the electron's kinetic energies when the electron hits perpendicular to the niobium. Si of thickness $300 \mu \mathrm{m}$ was used, and a glass cover was set in front of the Si to fully simulate the Si $p-i-n$ diode. In this simulation, the $\mathrm{Si} p-i-n$ diode was set just behind the niobium with thickness $2.5 \mathrm{~mm}$ and detected the transmitted signal through the niobium. From this simulation, electrons with more than $5 \mathrm{MeV}$ kinetic energy created almost the same diode current as those with $5 \mathrm{MeV}$ kinetic energy. On the other hand, the diode current created by electrons with $1 \mathrm{MeV}$ kinetic energy is 40, 200, and $10^{5}$ times smaller than those with 2,3 , and $5 \mathrm{MeV}$ kinetic energy, respectively. The high-energy electrons reflected at the 4-5, 5-6, and 6-7 iris positions come from a field emission source with a very small distribution in the $\mathrm{rf}$ phase and have extremely good sensitivity to the Si $p-i-n$ diode compared with the detected signals near the 8-9 iris at a $330^{\circ}$ cavity angle, which are dominant in the distribution of the rf phase and have low kinetic energies of less than $1 \mathrm{MeV}$. This is considered to be the reason why the broad spots were clearly obtained in the x-ray mapping in Fig. 5.

Finally, the reason why the obtained x-ray profile has both sharp and broad spots in Fig. 15 can be summarized as follows. First, it is clearly understood that most of the electrons from the field emission hit the opposite side of the source position and produce a sharp spot of the x-ray mapping. In addition, a broad spot can be produced when the accelerated electrons coming from the field emission source interact with the irises. The clear x-ray profile

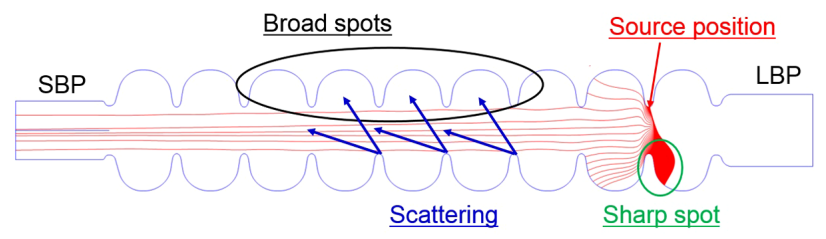

FIG. 15. Explanation of how the field emission creates radiation patterns seen in the experimental results of the $\pi$ mode of the fourth vertical test under $13.9 \mathrm{MV} / \mathrm{m}$ accelerating field with the No. 1 ERL cavity. 
together with appropriate mapping tools, such as our newly developed temperature and x-ray rotating mapping system, can be expected to identify some emitter positions with this detailed simulation. In addition, the measurement of the other passband mode of $7 / 9 \pi$ mode is very useful for identifying the source position of field emission for a multicell cavity with $\mathrm{x}$-ray mapping data.

\section{FIELD EMISSION STUDIES DURING CRYOMODULE OPERATION IN CERL}

We encountered degradation of the superconducting $\mathrm{rf}$ cavities in the cryomodule test and beam operation even though the performance of these cavities was good in the vertical test $[34,35]$. Field emission is the most important cause of this degradation. In particular, during the assembly work and beam operation, some dust and invisible particles might enter the cavity and generate field emission. It is crucially important not to degrade the cavity performance for the cryomodule operation during string assembly work on the cryomodule. In order to investigate the degradation due to the field emission of the cERL main linac cavities after the cryomodule assembly work and during the beam operation, we developed an appropriate $\mathrm{x}$-ray profile monitor not only for the vertical test but also for the cryomodule operation.

\section{A. Preparation for the field emission studies during the cryomodule operation}

The No. 3 and No. 4 ERL cavities were fabricated for installation in the cERL main linac cryomodule. Prior to the cryomodule assembly work, we conducted vertical tests on two cERL nine-cell cavities twice.

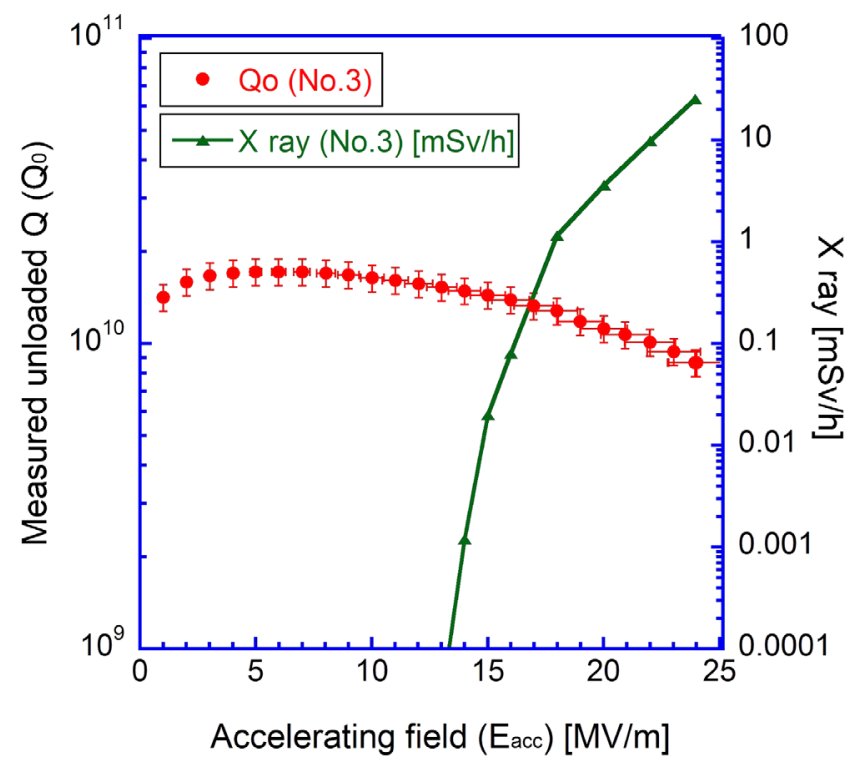

Figure 16 shows the results of the final vertical tests of No. 3 and No. 4 ERL cavities. They satisfied our requirements of a high unloaded $Q$ of more than $1 \times 10^{10}$ with an accelerating gradient of more than $15 \mathrm{MV} / \mathrm{m}$ [36]. The surface treatment and assembly work for these vertical tests were applied using the same procedure as that used for the No. 2 ERL cavity. Field emission started at 14 and $22 \mathrm{MV} / \mathrm{m}$ for the No. 3 and No. 4 ERL cavities, respectively, in both the final vertical tests. Field emission profiles were measured for all vertical tests by using the temperature and x-ray mapping system. However, it is impossible to use this mapping system in the cryomodule, because a helium jacket is mounted on the outside of the cavity. We need to consider another monitoring system. We knew that the field emission profile has an angular distribution not only just outside the cavity but also at both ends of the cavity sides, from the previous vertical tests and their simulation. We inferred that it would be possible to trace the change of the field emission sources by detecting the $\mathrm{x}$-ray angular distribution at just the helium jacket ends.

Figure 17 shows the setup of the $p-i-n$ diodes at the helium jacket ends (called the " $p-i-n$ profile monitor"). The $16 p-i-n$ diodes (HAMAMATSU, S1223-01) were arranged at every $22.5^{\circ}$ so as to surround each beam pipe near the end cell. We obtained a clear correlation between the $\mathrm{x}$-ray profile at the cavity cells obtained by the rotating mapping system and that of the $p-i-n$ profile monitors at both end sides. Figure 18 shows a typical example of the correlation. Figure 18 (top) shows the x-ray mapping data under a $22 \mathrm{MV} / \mathrm{m}$ accelerating field of the $\pi$ mode in the first vertical test of the No. 4 ERL cavity, and Fig. 18 (bottom left) shows the $\mathrm{x}$-ray profiles at the 1-2 iris and 8-9 iris positions for the same data. Figure 18 (bottom right) shows the

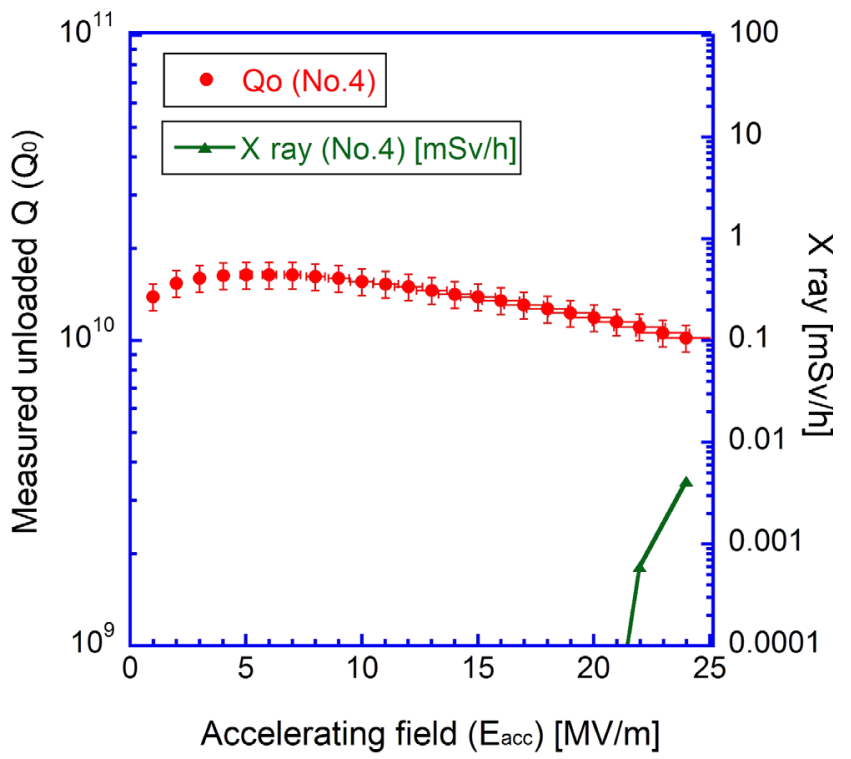

FIG. 16. Results of the final vertical test of the No. 3 ERL cavity (left) and No. 4 ERL cavity (right). The horizontal axis of each plot shows $E_{\text {acc }}$ of the measured accelerating field. The left (right) vertical axis of each plot shows the measured unloaded $Q$ (measured x-ray on the top flange). The dynamic range of the radiation monitor is from 0.0001 to $100 \mathrm{mSv} / \mathrm{h}$. 

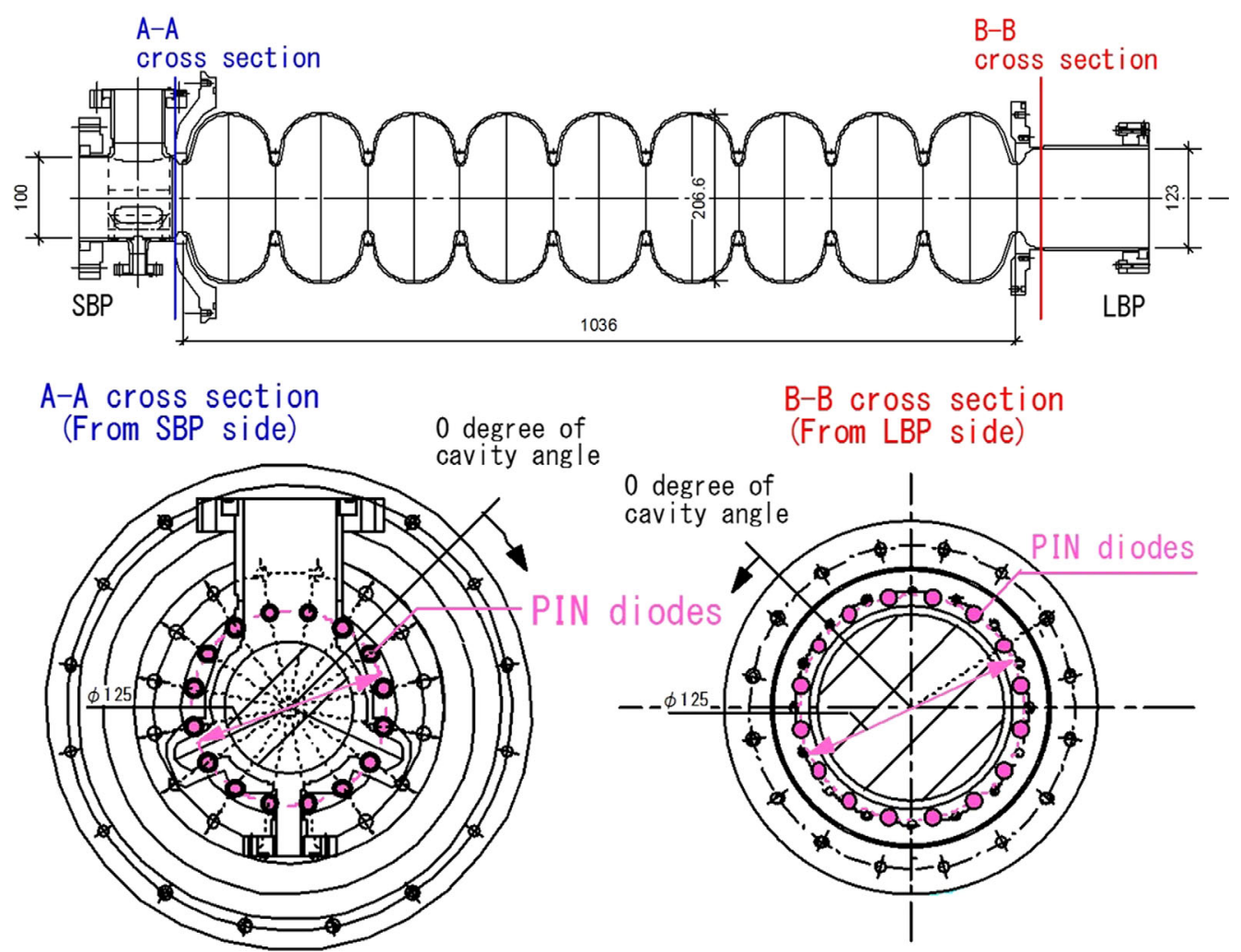

FIG. 17. Setup of the $p-i-n$ diodes at helium jacket ends. Pink circles show the setting position of the $p-i-n$ diodes. Thirty-two $p$ - $i$ - $n$ diodes were set to detect the angular distribution of $\mathrm{x}$-ray profiles of both sides.

measured x-ray angular profiles with both $p-i-n$ profile monitors. According to the x-ray mapping data, emitters would be expected to be located near the $0^{\circ}$ cavity angle and/ or the $180^{\circ}$ cavity angle. Therefore, we can expect that the electrons produced by emitters located at a $0^{\circ}$ cavity angle and/or a $180^{\circ}$ cavity angle would travel to the LBP side and produce these radiation patterns. The obtained $x$-ray profile with the $p-i-n$ profile monitor at the LBP side had large signals with broad distributions, which were also shown in the $x$-ray profile at the $8-9$ iris position located near the LBP side. On the other hand, that of the SBP side including the 1-2 iris positions had small signals. These measured $\mathrm{x}$-ray angular profiles with the $p-i-n$ profile monitors clearly revealed the same properties as the results obtained with the x-ray rotating mapping system from the standpoints of the broad spots on the LBP side with almost the same cavity angles and profile widths.

Figure 19 (top) shows the x-ray mapping data under a $22 \mathrm{MV} / \mathrm{m}$ accelerating field of the $\pi$ mode in the final vertical test of the No. 3 ERL cavity (left) and No. 4 ERL cavity (right), respectively. Figure 19 (bottom) shows the measured x-ray angular profiles with both $p-i-n$ profile monitors under a $22 \mathrm{MV} / \mathrm{m}$ accelerating field of the $\pi$ mode in the final vertical test of the No. 3 ERL cavity (left) and No. 4 ERL cavity (right), respectively. Similar correlations between the $\mathrm{x}$-ray angular profiles with the $p-i-n$ profile monitors and $\mathrm{x}$-ray profiles with the rotating mapping system were also observed in the final vertical tests of No. 3 cavity. There are no x-ray signals in the final vertical test of the No. 4 cavity.

These results indicate that these $p-i-n$ profile monitors are useful for identifying the field emission source from the angular distribution and for tracing the change in the cavity performance due to the field emission by comparing the $\mathrm{x}$-ray angular profiles in the vertical test to those in cryomodule operation. Figure 20 shows the measurement setup of the cryomodule with the $p-i-n$ profile monitors. The $p-i-n$ profile monitor has $16 p-i-n$ diodes (HAMAMATSU, S1223-01) arranged at the same angle as shown in Fig. 17. The $p-i-n$ profile monitors at the SBP sides surrounding the SBPs were cooled to $2 \mathrm{~K}$. The $p-i-n$ profile monitors at the LBP sides were set outside the cryomodule to surround the beam pipes beside the cryomodule, because the tuners occupied the space of the helium jacket ends at the LBP sides. Although the position of the $p-i-n$ profile monitors at the LBP sides seemed slightly farther from the cavity than in the original setup of the vertical test, the electrons traveling to the LBP sides due to the field emission were directly detected without any obstacles owing to the tapered beam pipe with a smaller diameter than the iris. The radiation doses were also detected with other radiation monitors (Hitachi Aloka Medical Ltd., MAR-782), which were the same monitors used in the vertical test. These radiation monitors were set 

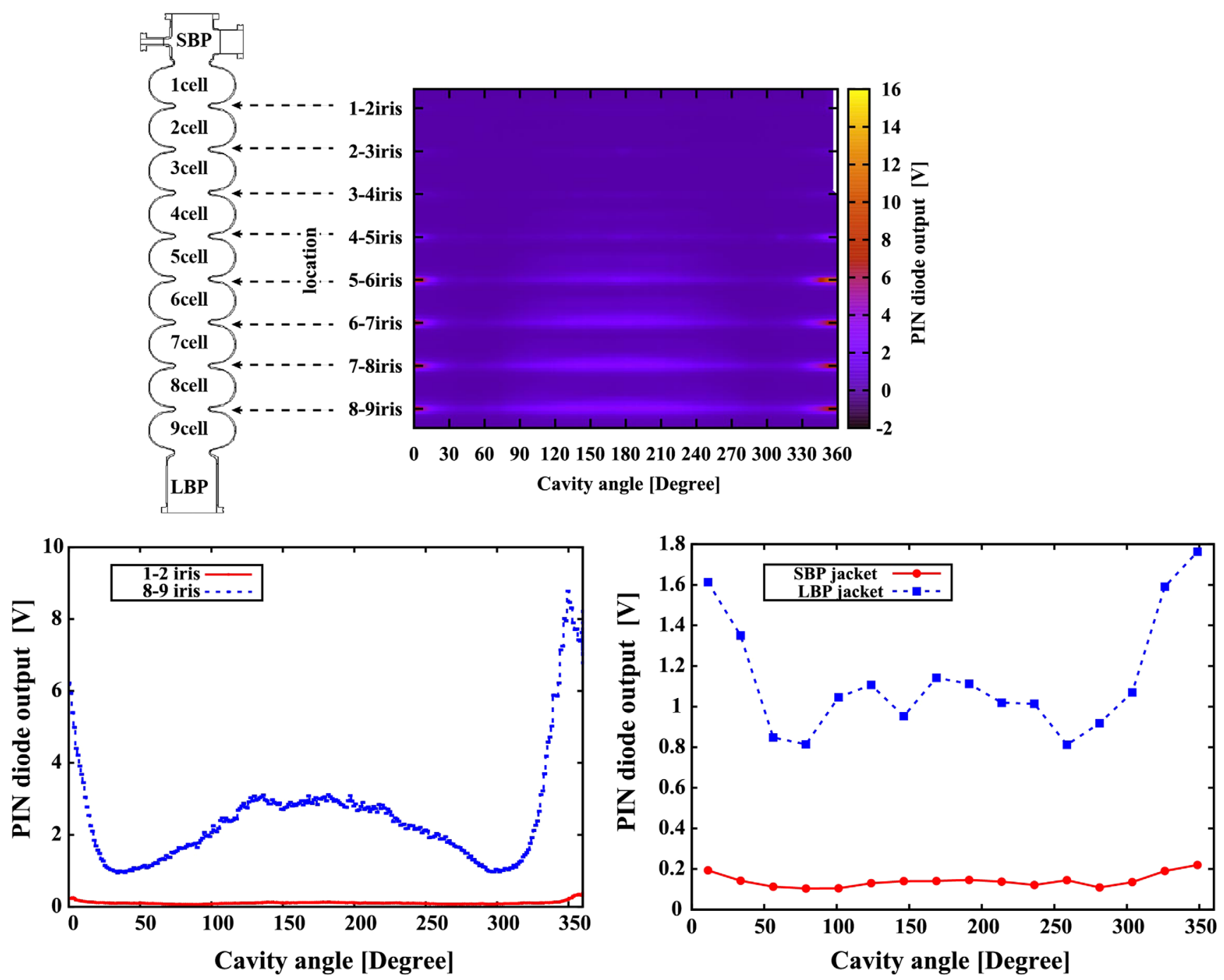

FIG. 18. (Top) Measured x-ray mapping data taken in the first vertical test of the No. 4 ERL cavity under a $22 \mathrm{MV} / \mathrm{m}$ accelerating field of the $\pi$ mode. (Bottom left) Measured x-ray profiles at the 1-2 iris and 8-9 iris locations of the top figure. (Bottom right) Measured $\mathrm{x}$-ray angular profiles with both the $p-i-n$ profile monitors. The horizontal axis shows the rotating angle of the mapping system. The vertical axis shows the voltage of the $p-i-n$ diode. Blue squares (red circles) show the measured voltage of each $p$ - $i$ - $n$ diode set at the LBP (SBP) side, respectively.

at both sides along the cavity axis outside the cryomodule more than $1 \mathrm{~m}$ away from the gate valve of the cryomodule (not shown in Fig. 20).

\section{B. Measurement results of ERL cavities with $p-i-n$ profile monitors during cryomodule operation}

We conducted a high-power test of the cERL main linac cryomodule. During the cryomodule operation, the accelerating field of only the $\pi$ mode in the cavities was excited. Figure 21 shows the measured unloaded $Q$ values $\left(Q_{o}\right)$ for both cavities. The $Q_{o}$ 's were estimated from the amount of liquid helium consumption of $P_{c}$ and the measured accelerating voltage of $V_{c}$ from the equation $Q_{o}=V_{c}^{2} /\left[(R / Q) \cdot P_{c}\right]$. The relationship between $V_{c}$, the cavity voltage, and $E_{\text {acc }}$, the accelerating field, is expressed as $V_{c}[\mathrm{MV}]=1.038[\mathrm{~m}] \times E_{\text {acc }}[\mathrm{MV} / \mathrm{m}] . V_{c}$ has almost the same value as the $E_{\text {acc }}$ of our cavities. The initially measured unloaded $Q$ value was higher than $1 \times 10^{10}$ near $15 \mathrm{MV} / \mathrm{m}$ and almost satisfied our requirements in the No. 4 ERL cavity. An unexpected radiation-increasing event decreased the unloaded $Q$ value. The degradation of $Q_{o}$ of the No. 3 ERL cavity was observed at an $E_{\text {acc }}$ higher than $10 \mathrm{MV} / \mathrm{m}$. Although $E_{\text {acc }}$ exceeded $15 \mathrm{MV} / \mathrm{m}$ for both the No. 3 and No. 4 ERL cavities without quenching, they both suffered from heavy field emission. The measured onsets were approximately $8 \mathrm{MV} / \mathrm{m}$ for both cavities [37,38].

We investigated the details of the degradation due to the assembly work after the vertical test and due to the unexpected radiation-increasing event of the No. 4 ERL cavity by checking the $\mathrm{x}$-ray angular profiles with the $p-i-n$ 

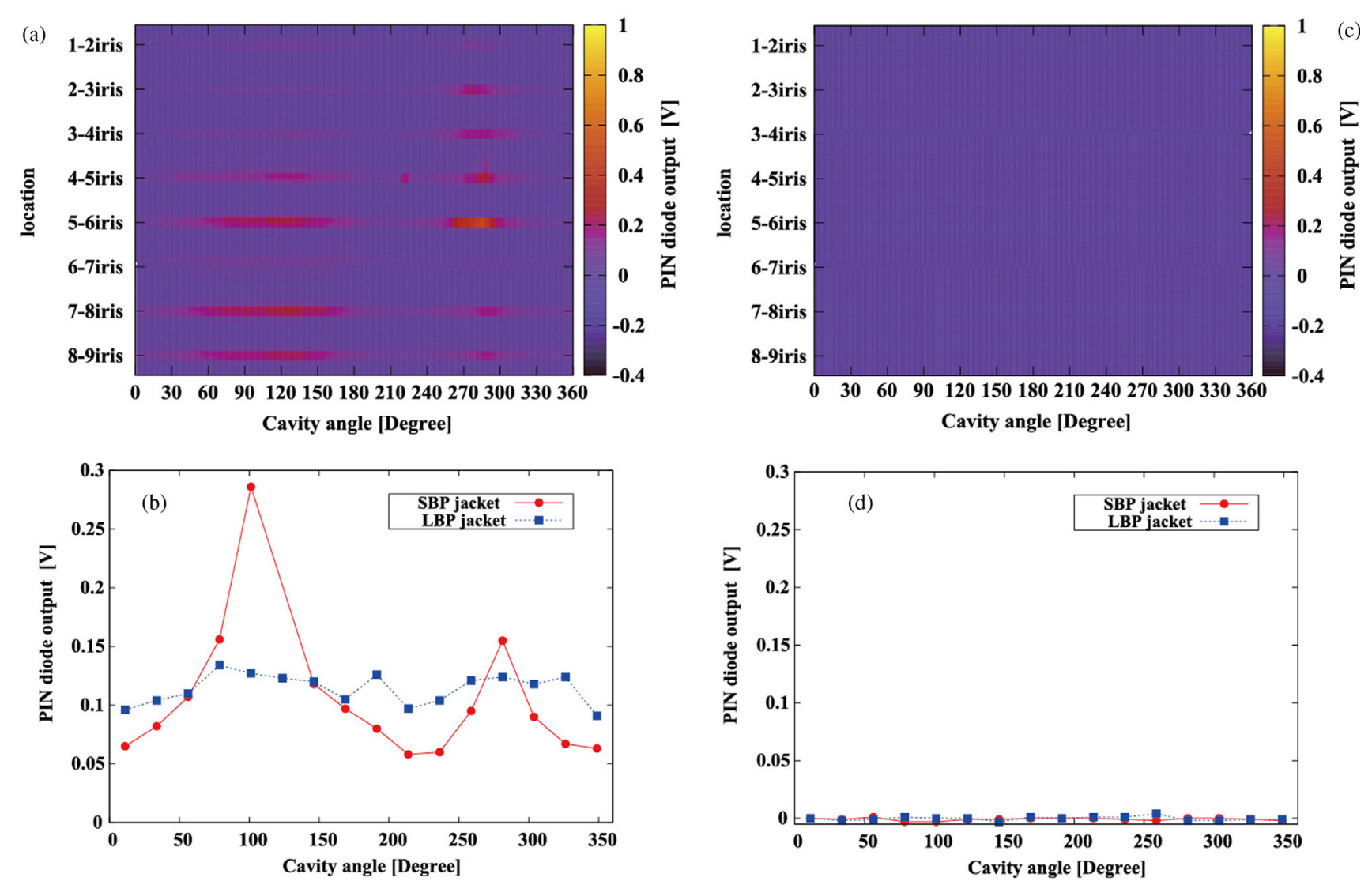

FIG. 19. (Top) Measured x-ray mapping data taken in the final vertical test of the No. 3 ERL cavity (a) and No. 4 ERL cavity (c) under a $22 \mathrm{MV} / \mathrm{m}$ accelerating field of the $\pi$ mode. (Bottom) Measured $\mathrm{x}$-ray angular profiles with both the $p$ - $i$ - $n$ profile monitors taken in the final vertical test of the No. 3 ERL cavity (b) and No. 4 ERL cavity (d) under a $22 \mathrm{MV} / \mathrm{m}$ accelerating field of the $\pi$ mode. The horizontal axis shows the rotating angle of the mapping system. The vertical axis shows the voltage of the $p$ - $i$ - $n$ diode. Blue squares (red circles) show the measured voltage of each $p-i$ - $n$ diode set at the LBP (SBP) side, respectively.

profile monitor during the cryomodule operation. Figure 22 shows the x-ray angular profiles during the cryomodule operation. As shown in Fig. 19, in the No. 3 ERL cavity, we found that the angular distribution changed from the final vertical test to the cryomodule test. In the No. 4 ERL cavity, we observed no $\mathrm{x}$-ray angular profile with the $p-i-n$ profile monitor in the final vertical test up to a $24 \mathrm{MV} / \mathrm{m}$ accelerating field as shown in Fig. 19. However, we clearly
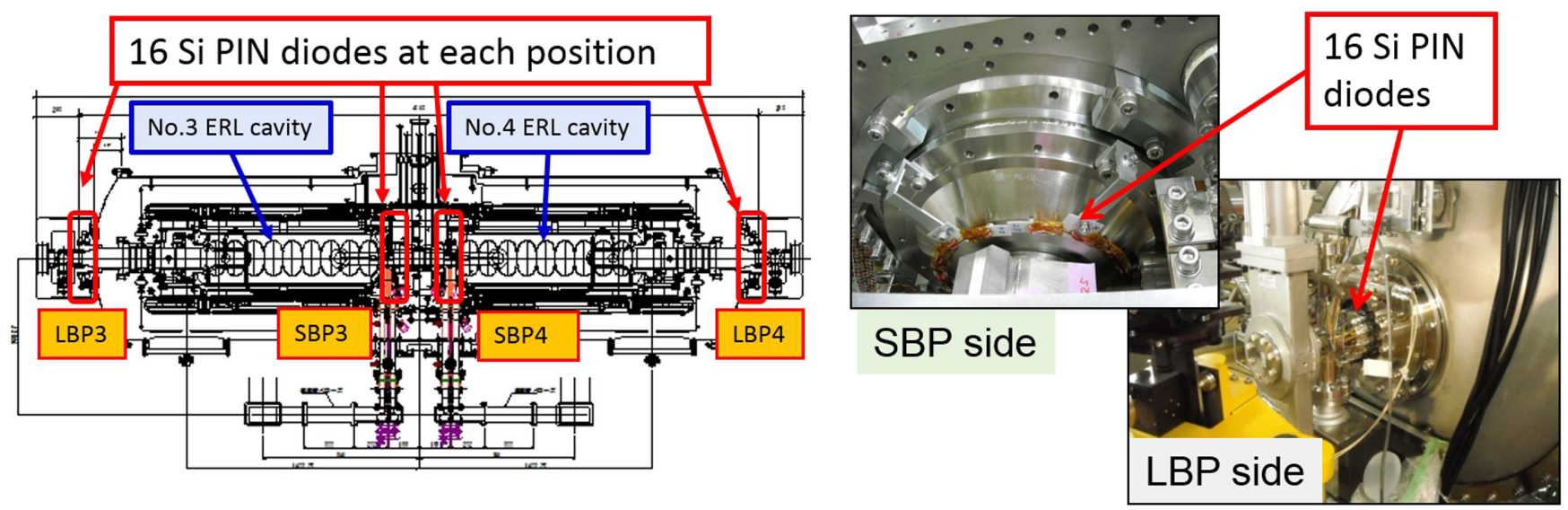

FIG. 20. (Left) Positions of the $p-i-n$ profile monitors of the cryomodule operation of the cERL main linac. The $p-i-n$ profile monitor had $16 p-i-n$ diodes, and four $p-i-n$ profile monitors were set at both ends of the No. 3 and No. 4 ERL cavities as shown in the red squares. (Right) Pictures of the $p-i-n$ profile monitors at the SBP side (upper) and LBP side (lower). 

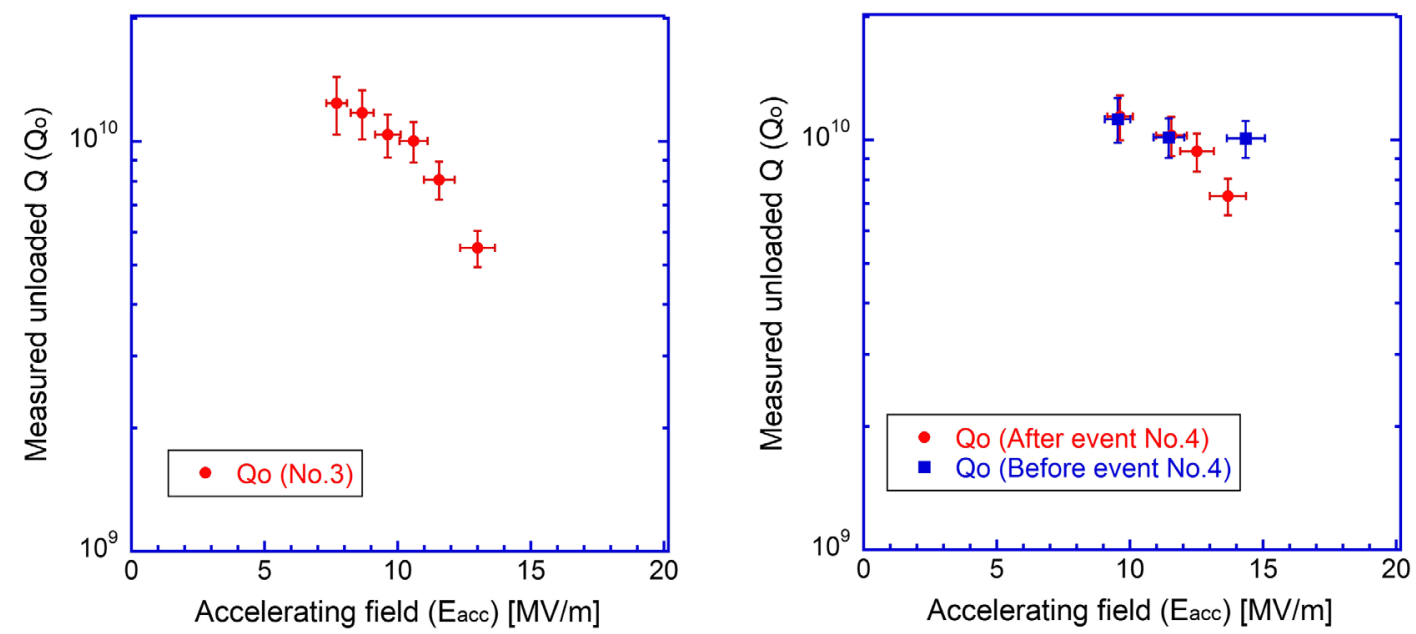

FIG. 21. Results of the high-power test of the No. 3 ERL cavity (left) and the No. 4 ERL cavity (right). The blue squares (red circles) in the right figure show the results before (after) the radiation-increasing event, respectively. The horizontal axis shows the accelerating field $\left(E_{\mathrm{acc}}\right)$, and the vertical axis shows the measured unloaded $Q$ in each plot.
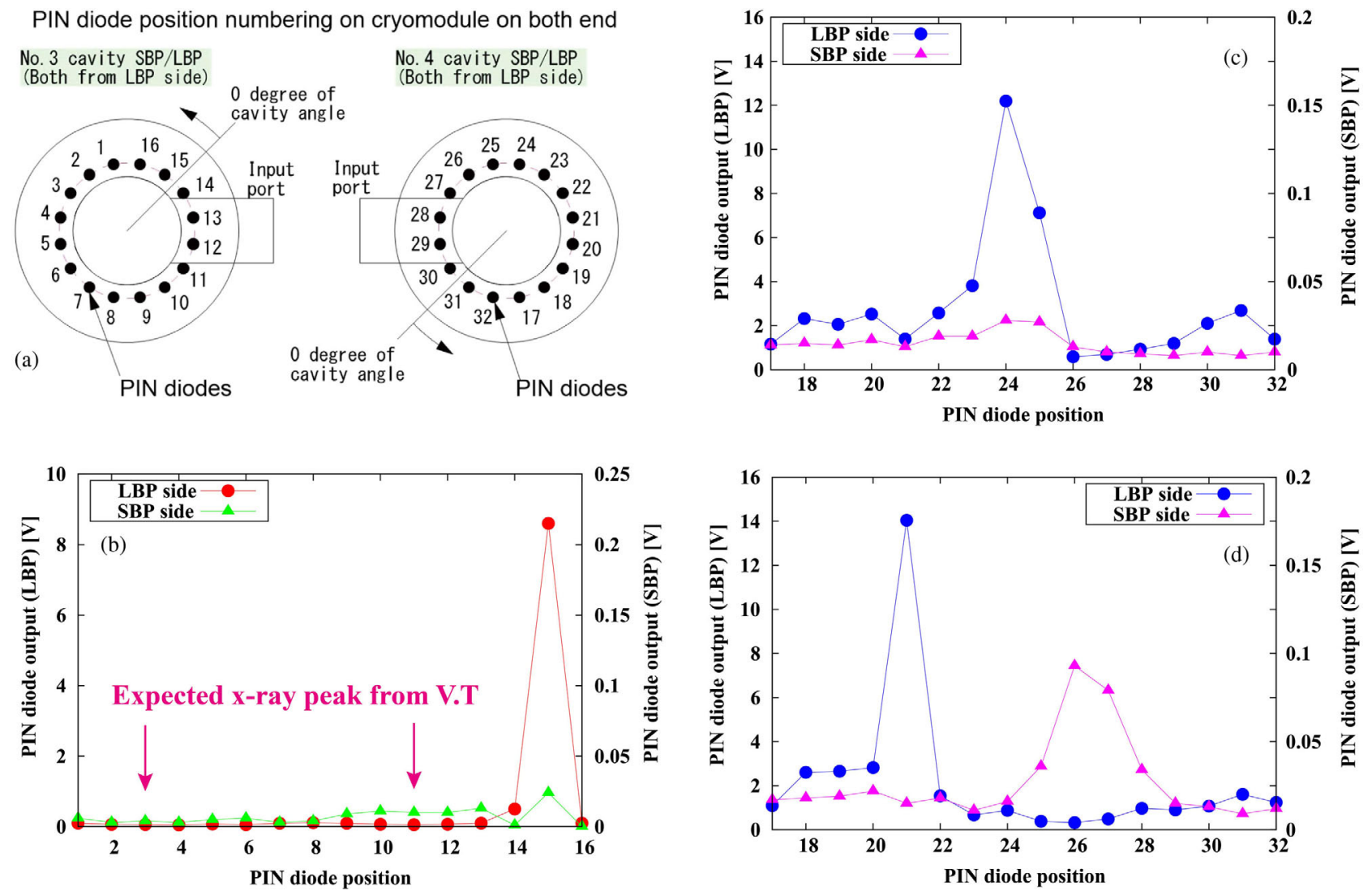

FIG. 22. (a) Arrangement of the $p-i-n$ diode position at the LBP and SBP sides on the cavity. Zero degree and the forward direction of the rotation of the cavity angle in this figure are the same as in Fig. 17. (b) The green triangles (red circles) of the x-ray angular profile with the $p-i-n$ profile monitor set on both ends of the No. 3 ERL cavity of the SBP (LBP) side under $9.6 \mathrm{MV} / \mathrm{m}$ during the high-power test, respectively. Pink arrows show the expected x-ray peak from the previous vertical test. (c),(d) The pink triangles (blue circles) of the $\mathrm{x}$-ray angular profile with the $p-i$ - $n$ profile monitors set on both ends of the No. 4 ERL cavity of the SBP (LBP) side during the highpower test under $13.5 \mathrm{MV} / \mathrm{m}$ before the unexpected radiation-increasing event (c) and under $11.5 \mathrm{MV} / \mathrm{m}$ after the unexpected radiation-increasing event (d), respectively. The horizontal axis of each plot shows the $p$ - $i$ - $n$ diode position in (a). The left and right vertical axes show the output voltages of the $p-i$ - $n$ diodes of the LBP and SBP sides, respectively. 


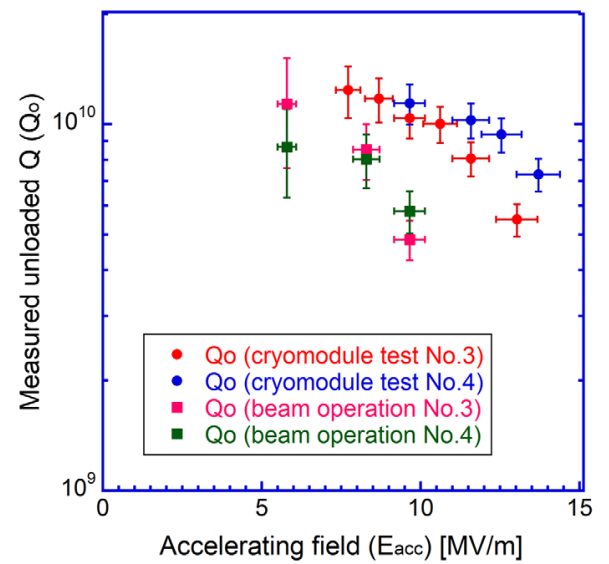

FIG. 23. Results of the measurements of the unloaded- $Q$ value of the No. 3 and No. 4 ERL cavities. The red (blue) circles show the final measurement results of the high-power test of the No. 3 (No. 4) ERL cavity as shown in Fig. 21. The pink (green) squares show the measurement results of the unloaded- $Q$ values of the No. 3 (No. 4) ERL cavity after the two-month beam operation in the cERL. The horizontal (vertical) axis shows the accelerating voltage (unloaded $Q$ ), respectively.
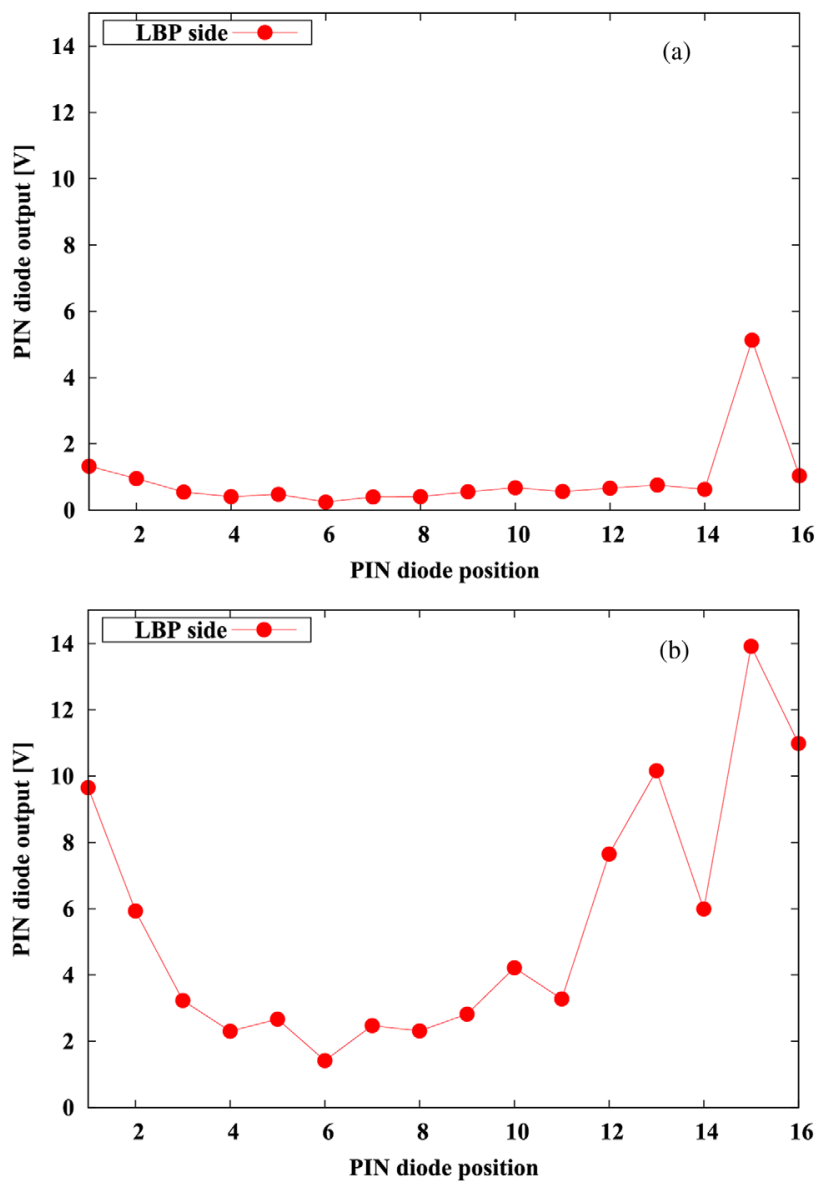

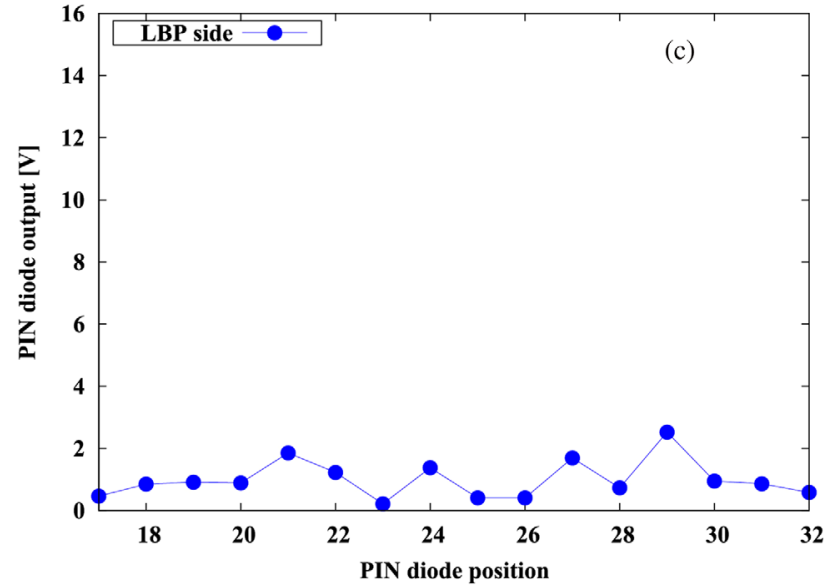

found the $\mathrm{x}$-ray angular profile during the cryomodule operation under a $14 \mathrm{MV} / \mathrm{m}$ accelerating field as shown in Fig. 22(c). We also found that the sudden unexpected radiation-increasing event significantly changed the angular distribution and substantially decreased the $Q_{o}$ during the cryomodule operation while maintaining $13.5 \mathrm{MV} / \mathrm{m}$ accelerating field of the No. 4 ERL cavity, as shown in Fig. 21 (right).

We continued the measurement of the $\mathrm{x}$-ray angular profiles with $p-i-n$ profile monitors during the beam operation of the cERL. Figure 23 shows the measurement results of the high-power tests before the beam operation and after the two-month beam operation of the cERL, respectively. We also encountered performance degradation during beam operation at both cavities. Figure 24 shows the X-ray angular profiles of the No. 3 and No. 4 ERL cavities at the LBP sides at the same time as above, respectively. We found that the two-month beam operation increased the $\mathrm{x}$-ray signals and changed the angular profiles on both LBP sides as the cavity performance was degraded.

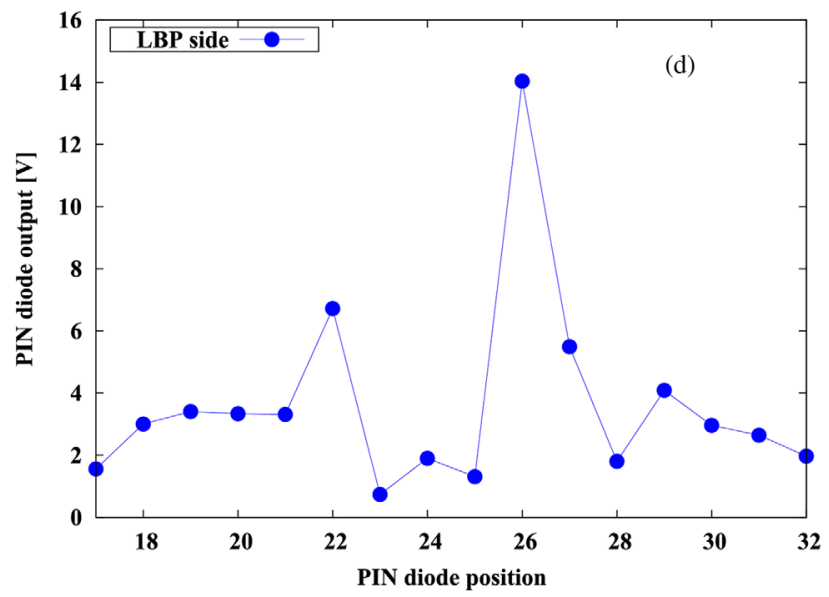

FIG. 24. Measured x-ray angular profile at the No. 3 ERL cavity before beam operation (a) and after two-month beam operation (b). Measured x-ray angular profile of the No. 4 ERL cavity before beam operation (c) and after two month beam operation (d). All angular profiles were obtained under a $9.6 \mathrm{MV} / \mathrm{m}$ accelerating field with the $p-i-n$ profile monitors at the LBP sides. The horizontal (vertical) axis shows the $p-i-n$ diode position (the measured voltages of the $p-i-n$ diodes) around the cavity, as shown in Fig. 22(a). 
TABLE IV. Summary of measured peak angles of $x$-ray angular profiles with $p$ - $i$ - $n$ profile monitor.

\begin{tabular}{|c|c|c|}
\hline \multirow[b]{2}{*}{ Cavity number (condition) } & \multicolumn{2}{|c|}{ Peak angle of x-ray angular profile } \\
\hline & Vertical test & High-power test at cryomodule \\
\hline No. 3 ERL cavity & $101^{\circ} \pm 11^{\circ}\left(281^{\circ} \pm 11^{\circ}\right)$ & $11^{\circ} \pm 11^{\circ}\left(191^{\circ} \pm 11^{\circ}\right)$ \\
\hline $\begin{array}{l}\text { No. } 4 \text { ERL cavity (before the unexpected } \\
\text { radiation-increasing event) }\end{array}$ & $\cdots$ & $214^{\circ} \pm 11^{\circ}\left(34^{\circ} \pm 11^{\circ}\right)$ \\
\hline $\begin{array}{l}\text { No. } 4 \text { ERL cavity (after the unexpected } \\
\text { radiation-increasing event) }\end{array}$ & $\cdots$ & $146^{\circ} \pm 11^{\circ}\left(326^{\circ} \pm 11^{\circ}\right)$ \\
\hline
\end{tabular}

\section{Discussion of field emission studies under cryomodule operation}

From the vertical tests, we demonstrated that the angular distribution of the $\mathrm{x}$-ray profile was precisely obtained with the rotating mapping system and that the $p-i-n$ profile monitor set at both ends gave us similar angular information to that obtained from the rotating mapping system described in Sec. VA. Therefore, the $p-i-n$ profile monitor enables us to compare the cryomodule operation with the vertical test in spite of fewer sensors. Table IV summarizes the measured cavity angles of the x-ray peaks obtained with the $p-i-n$ profile monitors. The measured angle was defined by the same setup of the rotating mapping system used in the vertical test, and the parentheses in Table IV show the opposite angle of the measured x-ray peak. In Sec. IV, we suggested that the source of the field emission is located at the opposite angle position of the measured x-ray peak. Even if we assume that the source of the field emission is not only at the measured x-ray peak angle but also at the opposite angle, the peak angles changed clearly before and after the vertical test. In addition, the unexpected radiation-increasing event also changed the peak angles. These results indicate that the sources of the field emission of both cavities were created during the string assembly work before the cryomodule operation and that the unknown emitter of field emission is created during this unexpected event. We could clearly distinguish the field emission sources associated with this unexpected event with the $p-i-n$ profile monitor set at both end sides at the LBP and SBP. Furthermore, during the long-term beam operation, we could observe an increase of the $\mathrm{x}$-ray signals with the $p-i-n$ profile monitors and degradation of the cavity performance. We supposed that the field emission sources created during the beam operation would increase the field emission so much as to degrade the cavity performance.

The $p-i-n$ profile monitors can provide important information about the degradation of the cavity performance due to field emission. During the two-month beam operation, we observed a sudden change of the $p-i-n$ profile monitor. Figure 25 shows the typical trend with the sudden change of the $p-i-n$ profile monitor of both LBP sides during beam operation for one hour. We kept the accelerating field at
8.3 MV $/ \mathrm{m}$, which is the nominal value of beam operation of the cERL main linac, for both No. 3 and No. 4 cavities. We encountered two radiation-increasing events, as shown in Fig. 25; one event occurred near the No. 4 cavity at about 20:00 and another occurred near the No. 3 cavity at about 20:30. Throughout these two events, the accelerating fields were kept constant. We also noted that there was no change of vacuum in the cavities on these two events and the effect of beam current was negligible for increasing radiation because of a very small beam current for this one hour. During the two-month beam operation, we sometimes observed similar radiation-increasing events, as shown in Fig. 25. Although we are not sure why these events occurred, it was clearly observed that the profiles of these $p-i-n$ profile monitors changed, as shown in Fig. 24, after the accumulation of these sudden radiation-increasing events, like Fig. 25, during the two-month beam operation. It is considered that the field emission would increase through these radiation-increasing events for two-month beam operation, and finally the cavity performance would degrade as shown in Fig. 23. When the profile changed and the signals of the $p-i-n$ profile monitors increased, we sometimes added a higher pulsed peak rf voltage, a technique called "pulse processing," to eliminate the field emission source. In our case, a few milliseconds of pulsed rf power was added to obtain a few $\mathrm{MV} / \mathrm{m}$ pulse in addition to the original accelerating voltage. We note that the peak of the high voltage of pulse processing is shorter than $0.1 \mathrm{~ms}$ because of the long rise time of the superconducting cavities. Because the pulse processing could decrease some signals of the $p-i-n$ diode, processing decreased some [39]. After the pulse processing, the cavity performance slightly recovered. Monitoring the x-ray angular profile with the $p-i-n$ profile monitors helps us maintain cavity performance.

Finally, we mention that the $p-i-n$ profile monitors were irradiated by the large radiation from the field emission under the long-term beam operation. In our case, the longterm irradiation by $\mathrm{x}$ ray from the field emission increased the offsets of the output voltage at some of the $p-i-n$ diodes without the cavity voltage. This offset must be subtracted from the obtained signal to measure the genuine $\mathrm{x}$-ray profile after the long-term beam operation. For short-term 

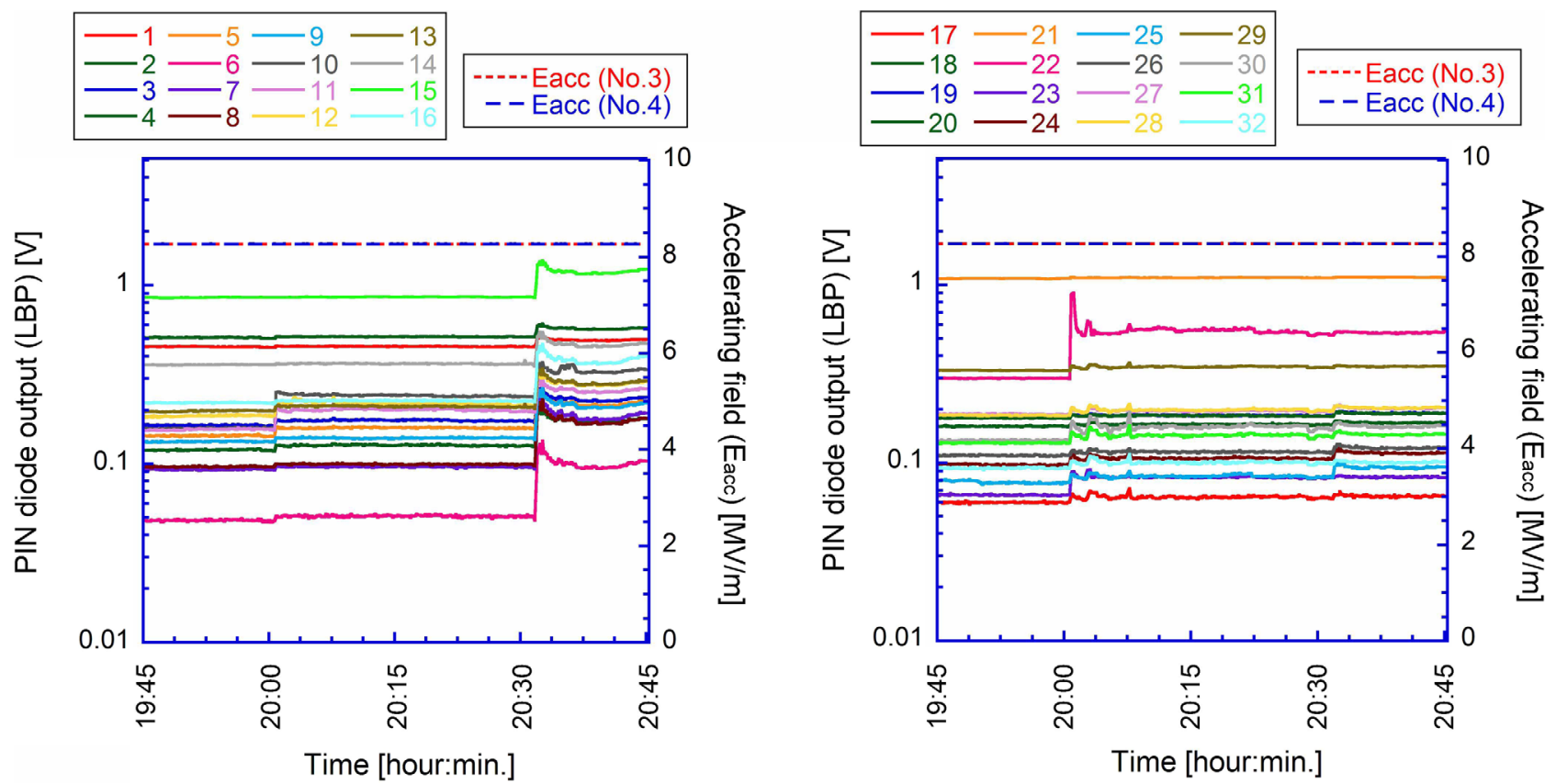

FIG. 25. The left (right) figure shows the time trend of the measured $p-i$ - $n$ diode output at the No. 3 (No. 4) cavity, respectively. The horizontal axis of each figure shows the time axis. The left (right) axis of each figure show the $p$ - $i$ - $n$ diode output (accelerating field), respectively. The solid lines of each figure show the $p-i-n$ diode output of each $p-i-n$ diode position at the LBP side around the cavity as denoted in Fig. 22(a). The red (blue) dotted lines show the accelerating field of the No. 3 (No. 4) cavity, respectively; these two dotted lines are superposed in the left and right figures.

irradiation, we could not observe the offset of the $p-i-n$ diode of the x-ray rotating mapping system under the vertical test.

\section{CONCLUSION}

In this paper, we described field emission studies conducted in the vertical tests and during cryomodule operation with the KEK-ERL model-2 cavities for the cERL. For precise field emission studies, we developed a new temperature and $\mathrm{x}$-ray rotating mapping system in the vertical test. Clear and precise temperature and $\mathrm{x}$-ray profiles around the nine-cell cavity with a high angular resolution of $0.5^{\circ}$ were observed with this mapping system. In the vertical test of the No. 1 ERL cavity, both broad spots and a local sharp spot on the x-ray mapping data were obtained in the $\pi$-mode measurement. On the other hand, only a local sharp spot on the x-ray mapping data was obtained in the other passband mode measurement. After the vertical test, the local tip on the iris position was observed with the inspection camera and located on the opposite cavity angle. These measured $\mathrm{x}$-ray profiles could be well explained with the calculation including both $\mathrm{rf}$ simulation and material interaction by assuming the observed local tip as the field emission source. In particular, these detailed simulations on field emission explained the mechanism for why the obtained local sharp spots of the $\mathrm{x}$-ray profiles were created in both the $\pi$ mode and the other passband mode. It is likely that the broad spot is created by reflected electrons at the iris. We also understood that the obtained x-ray local sharp spot was the result of the hitting of the field emission electrons, which also increased the temperature at the same local spot.

This deep understanding of the field emission mechanism in the vertical test with the temperature and x-ray rotating mapping system also led us to study the field emission in the cryomodule operation in detail. Although it is difficult to detect the X-ray profile of the cavity surrounded by a helium jacket in the cryomodule operation, we found that the angular distribution at the beam pipes contains some information about the field emission source based on our experience with vertical tests. We set the $p-i-n$ profile monitors on both end sides of the cavity to detect the angular profile of field emission. With this setup, we confirmed that the angular distribution of the $p-i-n$ profile monitor had a similar $\mathrm{x}$-ray profile to that obtained by the rotating mapping system in the vertical test. We also compared the cavity performance in the vertical test and in the cryomodule operation with the $p-i-n$ profile monitor. In particular, it enabled the change of the field emission sources during assembly work and during cryomodule operation to be traced by detecting the $\mathrm{x}$-ray angular profile due to field emission. We encountered performance degradation during cryomodule operation. However, by employing the measured $x$-ray angular profile with the $p-i-n$ profile monitor, we obtained a clearly different $\mathrm{x}$-ray 
angular pattern after the unexpected radiation-increasing event. It was found that this radiation-increasing event increased the field emission signals, causing degradation of the cavity performance. It is also effective to maintain the cavity performance during long-term beam operation, because we can detect the growth of the local field emission sources in the cavity by monitoring the angular distribution with the $p-i-n$ profile monitors.

Both the newly developed rotating mapping system for the nine-cell cavity in the vertical test and the $p-i-n$ profile monitors can give important information about the field emission from the radiation angular profiles. This information will aid recovery of the cavity performance and keeping the cavity performance stable during cryomodule operation. For example, we could recover cavity performance, which suffered from the increase of the field emission during the long-term cryomodule operation of the main linac in the cERL, by sometimes applying pulse processing safely while monitoring the change and decreasing the $p-i-n$ diode signal. Furthermore, the rotating mapping system allowed us to clearly identify the field emission source with the obtained temperature and $\mathrm{x}$-ray mapping data with high angular resolution. We believe that this mapping system with our deep understanding about the field emission mechanism will enable the development of sophisticated assembly work and a new processing method to suppress the field emission source more effectively.

\section{ACKNOWLEDGMENTS}

First, we express our gratitude to all members of the cERL development group for their continuous support for the construction and operation of the cERL and for the valuable discussions with them regarding the cERL main linac cryomodule operation. We are also grateful to all members of the Superconducting Rf Test Facility (STF) in KEK for supporting the vertical tests of our cavities. It is our pleasure to thank Dr. W. D. Moeller for introducing field emission studies by using the mapping system at DESY. This research was supported by a Government (MEXT) Subsidy for Strengthening Nuclear Security and by the Quantum Beam Technology Program of the Japanese Ministry of Education, Culture, Sports, Science, and Technology (MEXT) and partially supported by JSPS Grant-in-Aid for Scientific Research (KAKENHI) Grant No. JP20340066. This research was also supported by research support funds from the director of the Photon Factory and the director of the Institute of Materials Structure Science in KEK.

[1] T. Behnke, J. Brau, B. Foster, J. Fuster, M. Harrison, J. Paterson, M. Peskin, M. Stanitzki, N.Walker, and H. Yamamoto, ILC Technical Design Report [Online].
Available: https://www.linearcollider.org/ILC/Publications/ Technical-Design-Report.

[2] The European X-Ray Free-Electron Laser Technical design report, DESY Report No. 2006-097 2006, http://xfel.desy .de/localfsExplorer_read?currentPath=/afs/desy.de/group/ xfel/wof/EPT/TDR/XFEL-TDR-final.pdf.

[3] J. N. Galayda, in Proceedings of the 27th Linear Accelerator Conference (LINAC2014), Geneva, 2014 (JACoW, Geneva, 2014), p. 404, paper TUIOA04, http://www.JACoW.org/.

[4] Energy Recovery Linac Conceptual Design Report, KEK Report No. 2012-4, 2012, http://pfwww.kek.jp/ERLoffice/ detabase/ERL_CDR_full_text.pdf.

[5] edited by R. Hajima, N. Nakamura, S. Sakanaka, and Y. Kobayashi, Design Study of the Compact ERL, KEK Report No. 2007-7, 2008, https://jopss.jaea.go.jp/pdfdata/ JAEAResearch-2008-032.pdf.

[6] M. Akemoto et al., Construction and commissioning of the compact energy-recovery linac at KEK, Nucl. Instrum. Method Phys. Res., Sect. A 877, 197 (2018).

[7] N. Nishimori, R. Nagai, S. Matsuba, R. Hajima, M. Yamamoto, T. Miyajima, Y. Honda, H. Iijima, M. Kuriki, and M. Kuwahara, Generation of a $500-\mathrm{keV}$ electron beam from a high voltage photoemission gun, Appl. Phys. Lett. 102, 234103 (2013).

[8] K. Watanabe, S. Noguchi, E. Kako, K. Umemori, and T. Shishido, Development of the superconducting if 2-cell cavity for cERL injector at KEK, Nucl. Instrum. Methods Phys. Res., Sect. A 714, 67 (2013).

[9] K. Umemori, T. Furuya, S. Sakanaka, T. Suwada, T. Takahashi, H. Sakai, K. Shinoe, and M. Sawamura, in Proceedings of the 4th Asian Particle Accelerator Conference, Indore, 2007 (RRCAT, Indore, India, 2007), p. 570, paper THC2MA03, http://www.JACoW.org/.

[10] R. W. Roeth, V. G. Kurakin, G. Mueller, H. Piel, J. Pouryamout, D. Reschke, and N. Tellman, in Proceedings of the 5th Workshop on RF Superconductivity (SRF1991), Hamburg, 1991 (JACoW, Geneva, 1991), p. 599, paper SRF91F01, http://www.JACoW.org/.

[11] J. Knobloch, H. Muller, and H. Padamsee, Design of a high speed, high resolution thermometry system for $1.5 \mathrm{GHz}$ superconducting radio frequency cavities, Rev. Sci. Instrum. 65, 3521 (1994).

[12] Y. Yamamoto, H. Hayano, E. Kako, S. Noguchi, T. Shishido, and K. Watanabe, Achieving high gradient performance of 9-cell cavities at KEK for the international linear collider, Nucl. Instrum. Methods Phys. Res., Sect. A 729, 589 (2013).

[13] Q. S. Shu, G. Deppe, W.-D. Moeller, M. Pekeler, D. Proch, D. Renken, P. Stein, C. Stolzenburg, T. Junquera, A. Caruetta, and M. Fouaidy, in Proceedings of the Particle Accelerator Conference, Dallas, TX, 1995 (IEEE, New York, 1995), p. 1639, paper TPP11, http://www.JACoW.org/.

[14] K. Umemori, T. Furuya, T. Takahashi, H. Sakai, K. Shinoe, and M. Sawamura, in Proceedings of the 11th European Particle Accelerator Conference, Genoa, 2008 (EPS-AG, Genoa, Italy, 2008), p. 925, paper MOPP159, http://www JACoW.org/.

[15] H. Sakai, K. Shinoe, T. Furuya, T. Takahashi, K. Umemori, and M. Sawamura, in Proceedings of the 11th European Particle Accelerator Conference, Genoa, 2008 (EPS-AG, 
Genoa, Italy, 2008), p. 907, paper MOPP153, http://www .JACoW.org/.

[16] E. Kako, H. Hayano, S. Noguchi, T. Shishido, K. Umemori, K. Watanabe, Y. Yamamoto, H. Sakai, K. Shinoe, S. I. Moon, and Q. J. Xu, in Proceedings of the 13th Workshop on RF Superconductivity (SRF2007), Beijing, 2007 (JACoW, Geneva, 2007), p. 453, paper WEP10, http:// www.JACoW.org/.

[17] M. Sawamura, T. Furuya, H. Sakai, T. Takahashi, K. Umemori, and K. Shinoe, Eccentric-fluted beam pipes to damp quadrupole higher-order modes, Phys. Rev. ST Accel. Beams 13, 022003 (2010).

[18] H. Sakai, T. Furuya, N. Nakamura, K. Umemori, K. Shinoe, M. Sawamura, and E. Cenni, in Proceedings of the 15th International Conference on RF Superconductivity (SRF2011), Chicago (JACoW, Geneva, 2011), p. 356, paper TUPO005, http://www.JACoW.org/.

[19] K. Enami, T. Furuya, H. Sakai, M. Sato, K. Shinoe, K. Umemori, S. Michizono, T. Miura, F. Qiu, D. Arakawa, M. Sawamura, and E. Cenni, in Proceedings of the 5th International Particle Accelerator Conference (IPAC2014), Dresden (JACoW, Geneva, 2014), p. 2534, paper WEPRI027, http://www.JACoW.org/.

[20] M. Sawamura, T. Furuya, H. Sakai, K. Umemori, K. Shinoe, and E. Cenni, in Proceedings of the 15th International Conference on RF Superconductivity (SRF2011), Chicago (JACoW, Geneva, 2011), p. 350, paper TUPO003, http:// www.JACoW.org/.

[21] H. Sakai, T. Furuya, T. Takahashi, S. Sakanaka, K. Umemori, K. Shinoe, and M. Sawamura, in Proceedings of the International Particle Accelerator Conference, Kyoto, Japan (ICR, Kyoto, 2010), p. 2950, paper WEPEC028, http://www.JACoW.org/.

[22] Y. Iwashita, Y. Tajima, and H. Hayano, Development of high resolution camera for observations of superconducting cavities, Phys. Rev. ST Accel. Beams 11, 093501 (2008).

[23] K. Watanabe, H. Hayano, E. Kako, S. Noguchi, T. Shishido, Y. Yamamoto, and Y. Iwashita, in Proceedings of the International Particle Accelerator Conference, Kyoto, Japan (ICR, Kyoto, 2010), p. 2965, paper WEPEC033, http://www.JACoW.org/.

[24] E. Cenni, T. Furuya, H. Sakai, K. Umemori, K. Shinoe, and M. Sawamura, in Proceedings of the 15th International Conference on RF Superconductivity (SRF2011), Chicago (JACoW, Geneva, 2011), p. 789, paper THPO034, http:// www.JACoW.org/.

[25] G. Wu and E. Donoghue, FishPact, http://code.google .com/p/fishpact/.

[26] J. Billen and L. Young, Poisson superfish, Technical Report No. LA-UR-96-1834, Los Alamos, 2000.
[27] E. Cenni, T. Furuya, H. Sakai, K. Umemori, K. Shinoe, M. Satoh, and M. Sawamura, in Proceedings of the 3rd International Particle Accelerator Conference, New Orleans, LA, 2012 (IEEE, Piscataway, NJ, 2012), p. 295, paper MOPPC070, http://www.JACoW.org/.

[28] E. Cenni, Doctoral thesis, Department of Accelerator Science, The Graduate University for Advanced Studies (SOKENDAI), 2013.

[29] R. H. Fowler and L. Nordheim, Electron emission in intense electric fields, Proc. R. Soc. A 119, 173 (1928).

[30] H. Padamsee, J. Knobloch, and T. Hays, RF Superconductivity for Accelerators, 2nd ed. (Wiley, New York, 1998).

[31] Y. Li, K. Liu, R. Geng, and A. Palczewski, in Proceedings of the 16th International Conference on RF Superconductivity (SRF2013), Paris (JACoW, Geneva, 2013), p. 392, paper TUIOA06, http://www.JACoW.org/.

[32] High Voltage Vacuum Insulation, edited by R. Latham (Academic, London, 1995).

[33] W. R. Nelson, H. Hirayama, and D. W. O. Rogers, SLAC Report No. SLAC-265, 1985.

[34] S. Mitsunobu, T. Furuya, S. Takano, and Y. Yamamoto, in Proceedings of the 13th Workshop on RF Superconductivity (SRF2007), Beijing, 2007 (JACoW, Geneva, 2007), p. 170, paper TUP23, http://www.JACoW.org/.

[35] C. E. Reece, M. Drury, M. G. Rao, and V. Nguyen-Tuong, in Proceedings of the Particle Accelerator Conference, Vancouver, BC, Canada, 1997 (IEEE, New York, 1997), p. 3105, http://www.JACoW.org/.

[36] K. Umemori, T. Furuya, H. Sakai, M. Satoh, K. Shinoe, M. Sawamura, and E. Cenni, in Proceedings of the 3rd International Particle Accelerator Conference, New Orleans, LA, 2012 (IEEE, Piscataway, NJ, 2012), p. 2227, paper WEPPC011, http://www.JACoW.org/.

[37] H. Sakai, K. Enami, T. Furuya, M. Satoh, K. Shinoe, K. Umemori, M. Sawamura, and E. Cenni, in Proceedings of the 16th International Conference on RF Superconductivity (SRF2013), Paris, 2013 (JACoW, Geneva, 2013), p. 849, paper THIOC02, http://www.JACoW.org/.

[38] E. Cenni, K. Enami, T. Furuya, H. Sakai, M. Satoh, K. Shinoe, K. Umemori, and M. Sawamura, in Proceedings of the 16th International Conference on $R F$ Superconductivity (SRF2013), Paris, 2013 (JACoW, Geneva, 2013), p. 678, paper TUP091, http://www JACoW.org/.

[39] H. Sakai, M. Egi, K. Enami, T. Furuya, S. Michizono, T. Miura, F. Qiu, K. Shinoe, K. Umemori, and M. Sawamura, in Proceedings of the 17th International Conference on RF Superconductivity (SRF2015), Whistler, 2015 (JACoW, Geneva, 2015), p. 592, paper TUPB021, http://www .JACoW.org/. 